Portland State University

PDXScholar

Dissertations and Theses

Dissertations and Theses

$1-1-2010$

\title{
Quasi Steady Capillary Corner Flow
}

John Alex Baker

Portland State University

Follow this and additional works at: https://pdxscholar.library.pdx.edu/open_access_etds

Let us know how access to this document benefits you.

Recommended Citation

Baker, John Alex, "Quasi Steady Capillary Corner Flow" (2010). Dissertations and Theses. Paper 139. https://doi.org/10.15760/etd.139

This Thesis is brought to you for free and open access. It has been accepted for inclusion in Dissertations and Theses by an authorized administrator of PDXScholar. Please contact us if we can make this document more accessible: pdxscholar@pdx.edu. 


\title{
Quasi Steady \\ Capillary Corner Flow
}

\author{
by \\ John Alex Baker
}

A thesis submitted in partial fulfillment of the requirements for the degree of

\author{
Master of Science \\ in \\ Mechanical Engineering
}

Thesis Committee:

Mark Weislogel, Chair

Raul Cal

Yongkang Chen

Derek Tretheway

Portland State University

2010 


\begin{abstract}
It is possible to drain slender containers filled with wetting liquids via capillary flows along the interior corners of the container. Usually the well established equations governing such flows demand numerical techniques. In the case of container draining unique boundary conditions resulting from local section geometry allow for a quasi-steady assumption and in turn permit analytical solutions. The quasi-steady assumption may also be employed for certain problems in which the corner flows cause passive capillary migration of the fluid within the container. The analytic solutions are useful because of the ease in which geometric effects may be observed. Container draining and capillary migration by means of corner flows are studied in a variety of container geometries. It is shown that careful selection of cross sectional shape can be used to maximize drain rates and minimize capillary migration times. Three-dimensional effects for these flows are investigated in tapering containers. Some simple micro-scale experiments are reported that provide confidence in the assumptions and application of the important boundary conditions that enable the solutions.
\end{abstract}




\section{Acknowledgements}

I would like to thank Mark Weislogel for facilitating financial support, presenting the subject of the thesis to me, helping me understand all background material required and providing significant insight into the solutions and solution methods.

Ben Semerjian deserves credit for all kinds of help, including designing, machining and performing an experiment.

Curtis Brandsmith is noted for performing experiments. 


\section{Contents}

$\begin{array}{ll}\text { Abstract } & \text { i }\end{array}$

Acknowledgements $\quad$ ii

List of Tables $\quad$ v

List of Figures $\quad$ vi

1 Introduction 1

1.1 Motivation . . . . . . . . . . . . . . . . . . 1

1.2 Review of capillary corner flow . . . . . . . . . . . . . . 2

1.3 Impact of present work . . . . . . . . . . . . . . . . 6

1.4 Qualitative description of sample flows . . . . . . . . . . 6

1.5 Boundary conditions for local flow . . . . . . . . . . . . . . 9

2 Container draining solutions 13

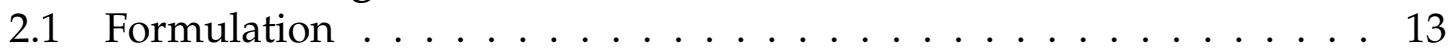

2.2 Constant section area . . . . . . . . . . . . . . 16

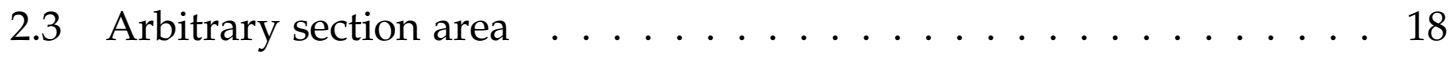

2.4 Imparted flow rates . . . . . . . . . . . . . . 20

3 Ullage migration solutions 23

3.1 Arbitrary section area . . . . . . . . . . . . . 23

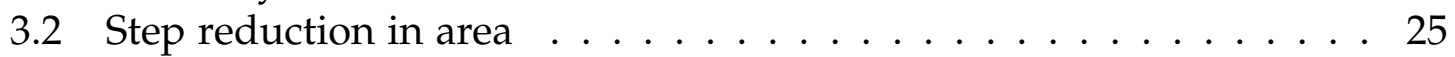

3.3 Imparted flow rates . . . . . . . . . . . . . . . 27

4 Container draining problems with relaxed constraints 31

4.1 Irregular cross sections . . . . . . . . . . . . . . 31

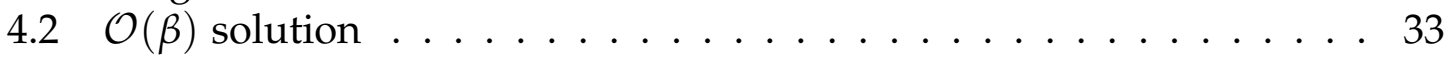

5 Quick reference guide 37

5.1 Dimensional solutions . . . . . . . . . . . . . . 37

5.2 Calculation of $F_{H}$ and $F_{Q}$ for some sample shapes . . . . . . . . 40

$\begin{array}{lll}6 & \text { Experiments } & 43\end{array}$ 
7 Conclusion $\quad 50$

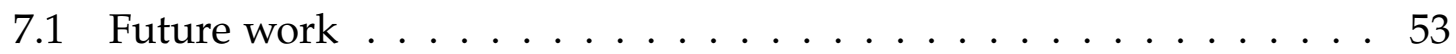

$\begin{array}{ll}\text { References } & 55\end{array}$ 


\section{List of Tables}

5.1 Dimensional Solutions . . . . . . . . . . . . . . . . . . . . . . . . . . 39

5.2 Volume Constraints . . . . . . . . . . . . . . . . . . 40 


\section{List of Figures}

1.1 Local flow schematic . . . . . . . . . . . . . . . . . . 4

1.2 Slender container partially filled with a wetting liquid . . . . . 7

1.3 Sketch of draining and ullage migration problem . . . . . . . 8

1.4 Local flow schematic for draining problem . . . . . . . . . . . 9

1.5 Schematic of constant height condition . . . . . . . . . 10

1.6 Flat bulk meniscus assumption . . . . . . . . . . . . . . 11

2.1 General section area local schematic, draining . . . . . . . . . . 14

2.2 Draining flow with imparted flow rate . . . . . . . . . . . . . 20

2.3 Solutions to a forced draining problem . . . . . . . . . 22

3.1 Ullage migration schematic . . . . . . . . . . . . . . . . . 24

3.2 Ullage migration stepped geometry . . . . . . . . . . . . 26

3.3 Ullage migration in stepped geometry with imparted flow rates . . . 28

3.4 Stepped ullage migration solutions . . . . . . . . . . . 30

5.1 Snow cone section . . . . . . . . . . . . . . . . . . . 41

5.2 Radially vaned cross section . . . . . . . . . . . . . . . 42

6.1 Experimental Setup . . . . . . . . . . . . . . . . . . . 44

6.2 The single draining experiment compared to theory . . . . . . . . 47

6.3 Scaled bulk meniscus overlays . . . . . . . . . . . . . 48

6.4 Experimental error . . . . . . . . . . . . . . . . . . . 49 


\section{Chapter 1}

\section{Introduction}

\subsection{Motivation}

Analyzing the behavior of fluids in space reveals challenges not usually present in terrestrial environments where the effects of surface tension are often negligible. At reduced gravity, surface tension forces must be accounted for even in large ( $1 \mathrm{~m})$ fluidic systems, making fluid behavior more difficult to predict. For example, when transferring fluid from one tank to another on earth it is standard to fill from the bottom of the tank and vent gas from the top. In space however spontaneous capillary flows may locate fluid over the vent, thereby venting the valuable fuel instead of gas [1]. Other routine processes on earth such as mass gauging, liquid acquisition, and even storage become more difficult in a low gravity environment [2]. In some cases fluid management can be critical, as was demonstrated when the Atlas Centaur flight AC-4 tumbled out of control due to inadequate settling of liquid hydrogen during venting [3].

Fluid behavior in low gravity is strongly influenced by container geometry [4] and even small changes may have drastic effects. This was unexpectedly demonstrated in a recent experiment where a small container asymmetry caused fluid to unexpectedly shift entirely to one side of a container [5]. It is shown in [6] that under identical conditions a triangular section has 64 times the capillary pumping capacity of a hexagonal section. Similar comparisons for critical heat inputs have been made for heat pipes utilizing capillary flows as a pumping mechanism [7]. 
Interior corners often have been employed in container designs to take advantage of this potential to passively control fluid behavior. For example, in the event of a sudden deceleration following thruster firing in spacecraft, interior corners act to quickly and spontaneously pump fluid within a fluid container, thereby reducing the overall settling time [8]. Lately, a urine processor has been designed for use aboard the International Space Station where an interior corner is employed to collect and pump urine into a collector for processing [9]. Capillary corner flows are utilized in terrestrial systems as well. Examples include micro-heat pipes [10]- [11] where it has been identified that performance is strongly related to the magnitude of the capillary pumping in corners [7], capillary filling of microstructures [12], water removal from fuel cells [13], hydrocarbon drainage [14] and microporous wicking structures [15]. Often in these works steady solutions to the local corner flow problems were employed, even if the flow was truly transient. This thesis provides analytical tools for studying capillary systems with transient corner flows by using a quasi-steady assumption. This assumption is detailed and validated in the sections to come.

\subsection{Review of capillary corner flow}

Capillary flow in sharp corners in the absence of gravity has been studied on numerous occasions. Using a finite element technique [16]-[17], a dimensionless friction factor has been determined and tabulated from which the local average velocity and a governing equation for the interface profile is found. In [18] a unique scaling method clearly identified the viscous resistance and the capillary driving force. Due to this scaling the friction factor was shown to be a tightly bound function. In this section a brief summary of [18] is provided as it forms 
the basis for this thesis.

A schematic of a single capillary corner flow is shown in Fig. 1.1. The $z$ coordinate is collinear with the corner vertex and the 'height' of the corner flow $h$ is the shortest perpendicular distance from $z$ to the fluid interface. A single non-linear PDE governs $h$

$$
\frac{\partial h^{2}}{\partial t}=\mathcal{W} \frac{\partial}{\partial z}\left(h^{2} \frac{\partial h}{\partial z}\right)
$$

where

$$
\mathcal{W} \equiv \frac{\sigma}{\mu}\langle\bar{w}\rangle^{*} \frac{\sin ^{2} \alpha}{f} .
$$

$\mathcal{W}$ can be thought of as a capillary velocity scale dependent on the corner half angle $\alpha$, contact angle $\theta$, surface tension $\sigma$ and viscosity $\mu$. The dimensionless function $f(\theta, \alpha)$ is a surface curvature function

$$
f=\frac{\sin \alpha}{\cos \theta-\sin \alpha}
$$

determined from geometry such that $h=R / f$ where $R$ is the radius of curvature of the interface in the cross flow plane. The term $\langle\bar{w}\rangle^{*}$ (referred to as $F_{i}$ in [18], but in more recent works it's meaning has been expanded and the nomenclature $\langle\bar{w}\rangle^{*}$ has been adopted) is the friction factor and $1 / 8 \lesssim\left\langle\bar{w}^{*}\right\rangle \leq 1 / 6$ for all values of $\alpha$ and $\theta$. These tight bounds are a result of the scaling that identifies the viscous resistance $\left(1 / \sin ^{2} \alpha\right)$ and the capillary driving force $(1 / f)$. A detail of the scaling method used is available in [19]. In this thesis, as in other works, $\langle\bar{w}\rangle^{*}$ is treated as an $\mathcal{O}(1)$ constant. Eq. 1.1 is appropriate for use under the following assumptions: 

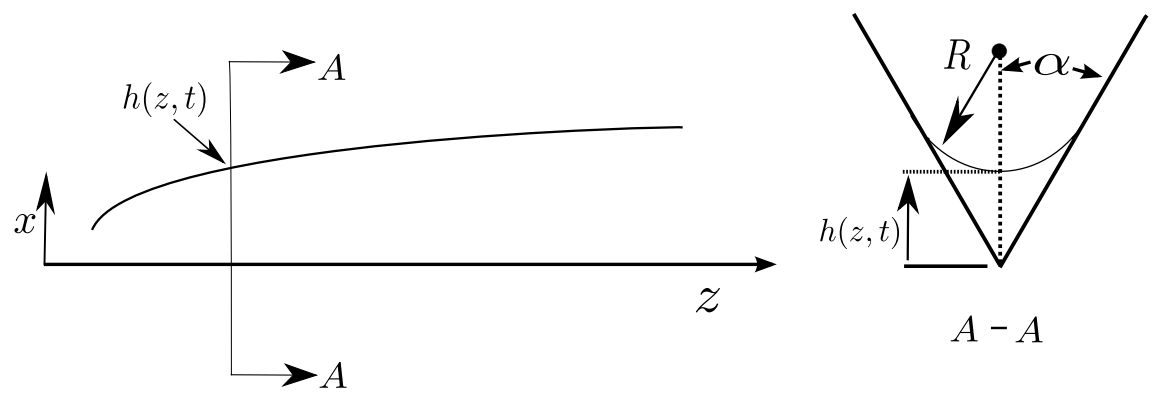

Figure 1.1: (left) Centerline meniscus profile of an individual corner flow. The height of the meniscus measured from the corner is $h(z, t)$. (right) A corner flow cross section. The meniscus is described by the radius of curvature $R$ and the corner half angle is $\alpha$.

- Gravity is negligible, requiring $B o \ll 1$. The bond number $\left(B o=\rho g L^{2} / \sigma\right)$ is a ratio of gravity to surface tension forces and is small for many common fluids on earth if the characteristic system dimension $L$ is $\leq \mathcal{O}(1 \mathrm{~mm})$. For space applications much larger systems are possible while maintaining $B o \ll 1$ due to the reduced gravity.

- The contact angle is the static equilibrium contact angle for the given liquid/gas/solid interface.

- The Concus-Finn wetting condition $\theta<\pi / 2-\alpha$ is satisfied [4].

- The fluid column is slender, requiring $\epsilon^{2} \ll 1$ where $\epsilon=h_{s} / z_{s}$ (the $s$ subscript denotes a 'scale' quantity). Both of these scales depend on the global geometry of the system in which the corner flows take place, and will be defined specifically later.

- The interface curvature is primarily in the cross flow plane, requiring $f \epsilon^{2} \ll$ 1. 
- The corner is perfectly sharp.

- The contact angle is identical on either side of the corner.

The remainder of this thesis utilizes all of the local assumptions above so that Eq. 1.1 is applicable throughout the corner flow domain. Other works have also utilized Eq. 1.1 to study capillary systems. In [20] networks of capillary corner flows were studied by applying novel boundary conditions to Eq. 1.1 and solving with a finite element method. The work in [6] provided closed form analytical solutions to Eq. 1.1 by identifying 2 global container geometries (a square pyramid and a wedge) that permit the quasi-steady assumption. For both containers, closed form zeroth order asymptotic solutions were found.

Some works have studied corner flows without making all of the assumptions listed above. For example, corner roundedness was considered in [21] which identified a 'narrow corner rectangular section' regime and a thin film regime. Gravity in the $x$ and $z$ (as depicted in Fig. 1.1) direction was taken into account in [22]. In these two works a governing PDE for the interface profile was given which can be reduced to Eq. 1.1 if the assumptions listed above were to be employed in an asymptotic sense.

In [23] asymmetrical wetting conditions were considered and a table of values for $\langle\bar{w}\rangle^{*}$ was presented depending on the contact angle on either side of the corner. Again in [24] similar tables were presented for the de-wetting regime in which the Concus-Finn condition was not met, as well as for 'gapped' corner geometries. In all of these works where an experiment was performed, the theory was shown to be valid within $\pm 6 \%$. 


\subsection{Impact of present work}

It is possible to solve Eq. 1.1 with a straightforward numerical routine, but analytical solutions are desired primarily because of the ease they offer in accounting for a myriad of geometries. As mentioned above, in [6] closed form analytical solutions were found for Eq. 1.1 in two geometries. This thesis builds on the ideas presented in [6] in two fundamental ways: (1) It is shown that not just a few, but a multitude of container geometries permit a quasi-steady assumption. The result is a system of equations which may be solved analytically in closed form. (2) A first order accurate solution is found in a simple geometry, giving confidence in solutions for more complicated container geometries as well as the constraints of the zeroth order solutions. Both of these points are demonstrated by studying sample capillary systems, and in doing so a framework solution method is established that can be applied to similar but more complicated capillary systems involving corner flows. The analysis is carried out by first determining the correct boundary conditions for the local corner flow Eq. 1.1, solving for the interface profile in terms of an unknown domain, then solving for the domain with a global mass balance. With the local flow characteristics and domain known, one can find other useful quantities pertaining to the capillary system, such as flow rates, meniscus velocities, and interface profiles.

\subsection{Qualitative description of sample flows}

A container partially filled with a wetting liquid in the presence of significant gravity is shown in Fig. 1.2. The fluid interface curves in order to meet the contact angle wetting condition at the container walls. This is most noticeable 


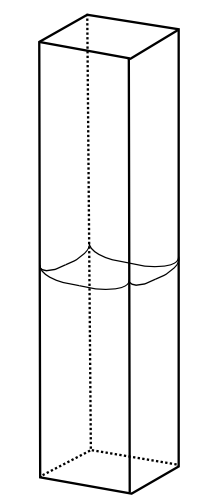

Figure 1.2: A slender container partially filled with a wetting liquid in 1-g (Bo》 1 ). Notice the radius of curvature of the liquid in the corner regions is slightly smaller than in the center of the interface.

in the corner regions of the container where the local radius of curvature is small. Gravitational forces become more significant away from the corners where the interface flattens. Because the pressure gradient is inversely proportional to the radius of curvature of the interface, it follows that the pressure is lower in the corner regions where the liquid rises. If gravity is suddenly and significantly reduced liquid will begin to rise in the corner regions to form a column due to capillary forces, eventually reaching the top of the container. This work considers two cases. The first case assumes a perfect sink exists such that all fluid reaching the top of the container is immediately removed. In this case, the corner flows act as pump and conduit draining the container of liquid. Such flows are referred to as 'draining flows' and an example is sketched in Fig. 1.3(a). Similar flows are found in heat pipe applications, where evaporation is the sink. A direct application in space is found when transferring fluid from one tank to another and capillary corner flows deliver fluid to the tank outlet (sink).

Case two examines flows in sealed containers that either taper or expand. 


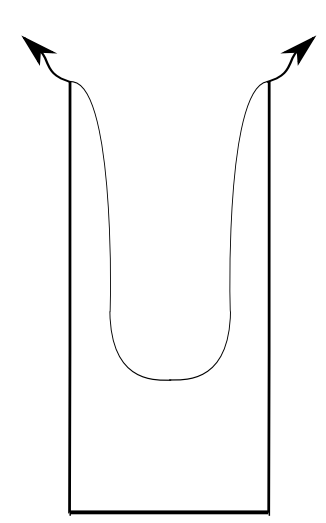

(a)

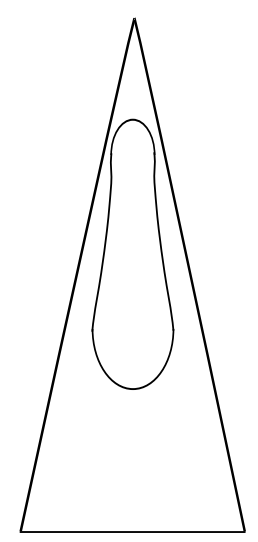

(b)

Figure 1.3: (a) 2-D sketch of a draining flow. The capillary corner flows pump fluid from the bulk region to the top of the container where fluid is being withdrawn (represented by the arrows). (b) An ullage migration problem. Corner flows pump fluid from the bottom of the container towards the top, displacing the gas ullage downward.

In this case the corner flows meet and form a new bulk meniscus that together with the existing bulk meniscus serve as book ends for a gas ullage as shown in Fig. 1.3(b). Liquid is then pumped along the corners from the bottom of the container to the top displacing the ullage toward the base of the container. These are referred to as 'ullage migration' problems. These may be encountered in space applications where fluid is re-distributed following sudden deceleration as well as in micro-scale applications where bubbles (perhaps undesirably) migrate within capillary tubes.

Both problems are studied analytically in this thesis. It will be shown that both of these types of flows (draining and ullage migration) permit a quasi-steady assumption. A variety of cross section shapes are considered, including regular and irregular polygons, 'snow cones', and radially vaned sections. Note that ullage migration problems were studied previously in [6] for pyramid and wedge 


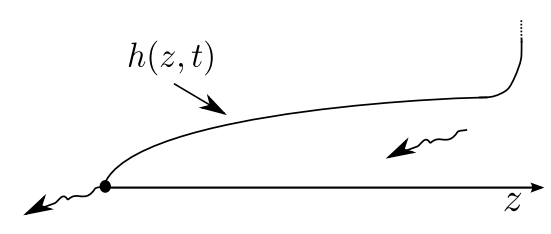

Figure 1.4: The centerline meniscus height of a single corner flow in a draining problem with a constant cross sectional area container. The $z$ coordinate is collinear with the corner and the origin is at the container outlet where fluid is withdrawn. Fluid is being pumped from the bulk region (right) to where it is withdrawn at $z=0$ (left).

shaped containers. In both cases variable cross sectional area containers are studied. Zeroth order solutions for the bulk meniscus positions as functions of time are provided for arbitrary section area and taper. These solutions are consistent with the pyramidal section result of [6]. Comparisons of analytical predictions to experiment show good agreement, thus validating the modeling assumptions.

\subsection{Boundary conditions for local flow}

A single corner flow in a draining problem with constant section properties is sketched in Fig. 1.4. As shown a capillary flow pumps liquid along the corner from the bulk to the tip where it is being withdrawn. Eq. 1.1 is used to find $h(z, t)$ and therefore two spatial boundary conditions are needed. The first is the sink condition at the tip, $h(0, t)=0$. Other constant boundary conditions can be utilized without significant complication, but the sink is used for clarity in presentation and because it yields a maximum flow rate condition [18]. For the second boundary condition it has been determined experimentally that $h$ achieves a known value near the bulk meniscus depending on the local cross sectional area 


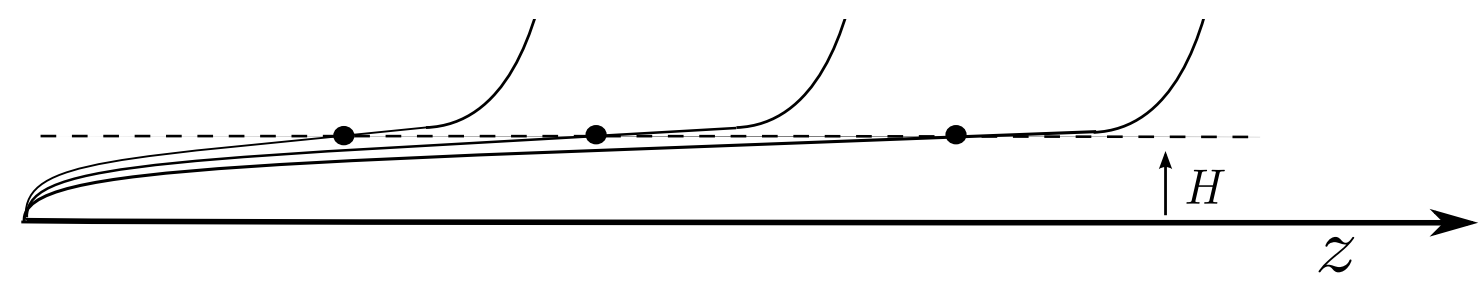

Figure 1.5: Three local interface profiles at different times for a container draining problem. Each profile has height $H$ near the bulk region.

$A_{s}$ and cross section shape. These observations are sketched in Fig. 1.5. The known height $H$ is computed by first determining the mean radius of curvature $R$ of the liquid interface if the entire container were infinitely long, had cross sectional properties identical to those found near the bulk meniscus (exactly how near will be addressed momentarily) and was in static equilibrium. The methods of de Lazzer et al. [25] are used to determine $R$. $H$ is then computed from the surface curvature function 1.2,

$$
H=\frac{R}{f} .
$$

This method for computing the local corner flow meniscus height near a bulk meniscus has been used for similar flows with good results [18], [26] and in [6] the method was applied to 'weakly 3-D' sections in which $R=R(z)$; the same procedure will be performed here. Some clear examples of computing $R$ can be found in [8] for polygonal sections, vaned containers, snow cone sections and others. For this work an important result from [25] is that cross section shapes in which the quantity $A_{s} / P_{s}^{2}\left(P_{s}\right.$ is the section perimeter) is independent $z$ an 

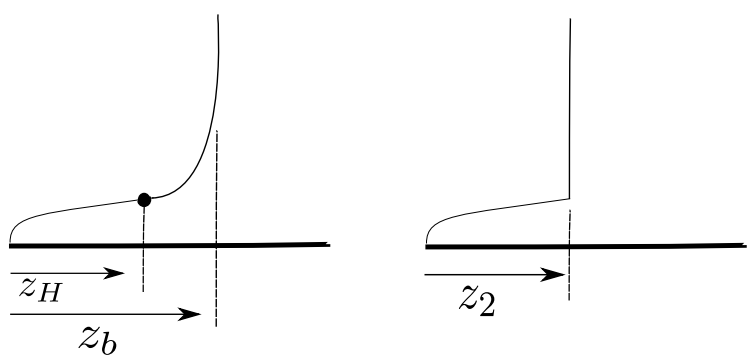

Figure 1.6: A sketch of a container draining problem interface on the left. The distance from the container outlet to the point at which the corner flow establishes height $H$ is $z_{H}$. The distance from the outlet to the center of the bulk meniscus is $z_{b}$. The model is drawn on the right which assumes $z_{H}=z_{b} \equiv z_{2}$.

equation for $R$ can be written as

$$
R=F_{R} A_{s}^{1 / 2}
$$

where $F_{R}$ is a dimensionless function of the cross sectional geometry. Many common shapes possess this property, including regular polygons, rhombi and snow cones. Through Eq. 1.3 a relatively compact equation can then be written for $H$

$$
H=F_{H} A_{s}^{1 / 2},
$$

where $F_{H}=F_{R} / f$. A few of the functional forms for $F_{H}$ are reported and briefly discussed in Chapter 5.

It is also necessary to determine where the corner flows establish height $H$, i.e. $h(z=$ ?,t $)=H$. With reference to Fig. 1.6 it is desirable to define the known height location $z_{H}$, in terms of the bulk meniscus position $z_{b}$. A simple modeling assumption is that the bulk region is small compared to the corner flow length 
such that $z_{H} \approx z_{b} \equiv z_{2}$, and that is what is made here. Past experiments have established confidence in this assumption, and further experimental evidence is provided in Chapter 6. Furthermore, it is assumed that the gas volume in the bulk region is small compared to the total volume. These two assumptions are employed in the model by assuming a flat bulk meniscus, depicted in Fig. 1.6 (right). This method of computing boundary conditions for the local corner flows near a bulk meniscus is employed throughout the thesis. It is noted that all of these solutions are enabled by this boundary condition, and the author is not aware of an alternative method that is equally reasonable and easy to employ. Due to the relative simplicity of the boundary condition, it is then possible to analyze three dimensional transient free surface problems using analytical methods, as demonstrated in the following sample problems. 


\section{Chapter 2}

\section{Container draining solutions}

In this chapter a system of equations governing the bulk meniscus position in container draining problems is derived, non-dimensionalized and solved asymptotically. Tapering and expanding sections are considered, as well as combined forced/capillary systems. The cross section shape is not defined explicitly, as cross section shape effects are absorbed in the scaling.

\subsection{Formulation}

In Fig. 2.1 a single corner flow and bulk meniscus are drawn for an arbitrary 'equi- $f$ ' geometry. The term 'equi- $f$ ' implies that the surface curvature function $f$ is the same for each corner, so that the boundary condition at the flat bulk meniscus is identical for each corner flow in the model. This is not necessary to yield solutions, but is assumed for clarity in presentation because then each corner flow has identical boundary conditions. This assumption is relaxed in 4 and the governing system of equations is presented. The length of the corner flow $z_{2}$ is measured along the local $z$ axis, and the bulk meniscus position $z_{2}^{\prime}$ is measured along a global coordinate $z^{\prime}$ located in the center of the container. The perpendicular distance from $z$ to $z^{\prime}$ is $\mathcal{S}\left(z^{\prime}\right)$ and the section area is defined in terms of $z^{\prime}$ such that

$$
A_{s}=A_{0} A_{s}^{*}
$$




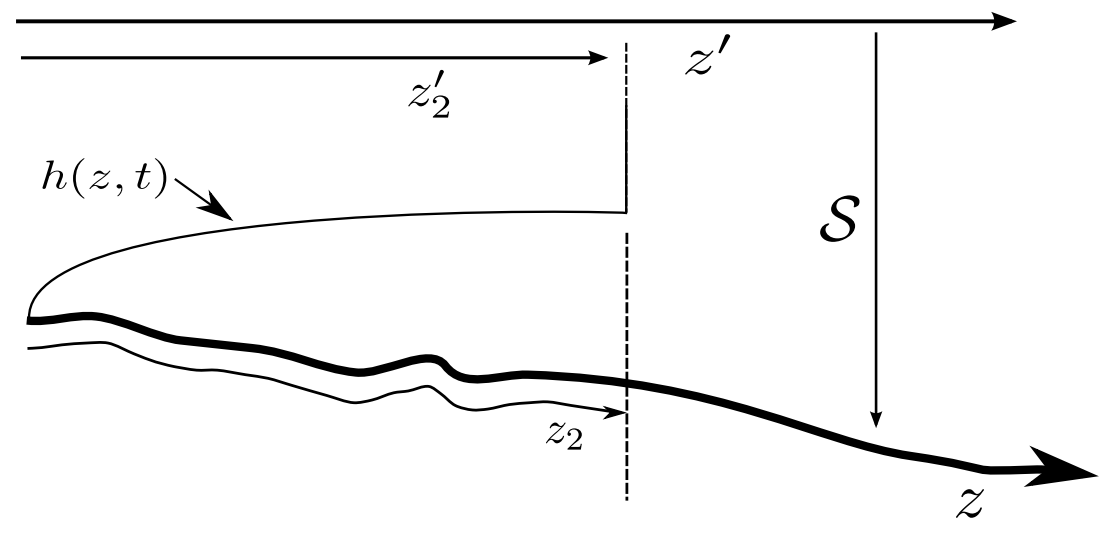

Figure 2.1: A schematic of a single corner flow in a draining problem with a cross sectional area (but not shape) that varies with $z$. The coordinate $z^{\prime}$ runs along the center of the container and $z$ runs along the corner. The perpendicular distance from $z^{\prime}$ to $z$ is $\mathcal{S}$. The subscript 2 refers to the position of the flat bulk meniscus.

where $A_{0}$ is a constant with dimensions of area and $A_{S}^{*}$ is a dimensionless section area function of $z^{\prime}$. For example, a container with a constant section area has $A_{s}^{*}=$ 1 while an expanding section may be defined such that say, $A_{s}^{*}=\left(1+z^{\prime} / z_{s}\right)^{2}$. In order to achieve analytical solutions it seems necessary to assume $z_{2} \approx z_{2}^{\prime}$ and the constraint $\left|\partial \mathcal{S} / \partial z^{\prime}\right|^{2} \ll 1$ is assumed, thereby defining a 'weakly' $z$-dependent cross sectional area in which $z_{2} \approx z_{2}^{\prime}$. Employing these assumptions the local corner flow Eq. 1.1 and boundary conditions

$$
h(0)=0 \quad h\left(z_{2}\right)=H\left(z_{2}\right)
$$

may be solved to find $h(z, t)$ in each wetted corner. With $h$ known the local capillary flow rate $Q_{c a p}$ can be found through

$$
Q_{c a p}=m \mathcal{W} F_{A} h^{2} \frac{\partial h}{\partial z}
$$


where $m$ is the number of wetted corners and $F_{A}(\alpha, \theta)$ is yet another geometric function defined such that $A_{f}=F_{A} h^{2}$ where $A_{f}$ is the flow area in a single corner,

$$
F_{A}=f^{2}\left(\frac{\cos \theta \sin \delta}{\sin \alpha}-\delta\right)
$$

and $\delta=\pi / 2-\alpha-\theta$ [18]. An integral mass balance can then be employed to determine $z_{2}$ by setting the flow rate of liquid leaving the container equal to the flow rate of gas entering the container,

$$
\left.Q_{c a p}\right|_{z=0}=\frac{\partial V}{\partial t}=\frac{\partial}{\partial t} \int_{0}^{z_{2}}\left(A_{s}-m F_{A} h^{2}\right) d z
$$

where $V$ is the volume of gas in the container at any given time. One initial condition is needed to solve 2.4. One choice is specification of the initial volume of gas in the container,

$$
V(0)=\int_{0}^{z_{2}(0)}\left(A_{s}-m F_{A} h^{2}\right) d z
$$

Note that in order to apply 2.5 it is necessary to first solve the corner flow problem, Eq. 1.1. In total, the local corner flow Eq. 1.1 and the mass balance Eq. 2.4 with the boundary conditions represents a system of two equations and two unknowns to be solved for the meniscus location $z_{2}$ in a container being drained by capillary corner flows. 


\subsection{Constant section area}

The analysis for draining flows in constant cross sectional area containers is simplified because the boundary condition for 1.1 at the bulk meniscus is independent of $z$ and the mass balance Eq. 2.4 loses a degree of complexity because $A_{s}$ may be pulled out of the integral. Because few closed form analytical solutions are known for the full governing equations 1.1 and 2.4, the entire system is non-dimensionalized to identify the essential parameters and limiting behavior. The scales chosen are

$$
z_{s}=V(0) / A_{0} \quad t_{s}=\frac{A_{0} z_{s}}{Q_{s}} \quad h_{s}=H .
$$

The term $Q_{s}$ should be thought of as a capillary flow rate scale $Q_{s}=m \mathcal{W} F_{A} H^{3} / z_{s}$. The length scale $z_{s}$ is arbitrary and divides out of all equations except the boundary condition for the global mass balance. The condition $z_{s}=V(0) / A_{0}$ is chosen for clarity of presentation and is approximately equal to the initial length of the corner flow, $z_{s} \sim z_{2}(0)$. The time scale is found by balancing the first and second terms of the global mass balance 2.4 and $h_{s}$ is chosen so that $h^{*}\left(z_{2}^{*}\right)=1$. Employing these scales gives the dimensionless local equation for each corner flow,

$$
\beta \frac{\partial h^{* 2}}{\partial t^{*}}=\frac{\partial}{\partial z^{*}}\left(h^{* 2} \frac{\partial h^{*}}{\partial z^{*}}\right),
$$

subject to $h^{*}(0)=0$ and $h^{*}\left(z_{2}^{*}\right)=1$. The global mass balance is

$$
\left.h^{* 2} \frac{\partial h^{*}}{\partial z^{*}}\right|_{0}=\frac{\partial}{\partial t^{*}} \int_{0}^{z_{2}^{*}}\left(1-\beta h^{* 2}\right) d z^{*}
$$


subject to $z_{2}^{*}(0)=1-\beta \int_{0}^{z_{2}^{*}(0)} h^{* 2} d z^{*}$ where $\beta$ is the ratio of corner flow area at $z_{2}$ to total cross sectional area, $\beta \equiv m F_{A} H^{2} / A_{s}$. More precisely though, $\beta$ should be thought of as a ratio of the local to global time scales, signalling the classic quasi-steady condition of a quickly developing local system interacting with a slowly developing global system, a situation that de-couples the governing equations at zeroth order. An analytical asymptotic solution is then pursued by assuming $\beta \ll 1$, and the zeroth order solution for 2.7 is

$$
h^{*}=\left(C_{1}+C_{2} z\right)^{1 / 3}
$$

with constants found by applying the boundary conditions. From 2.9 the dimensionless flow rate $Q_{\text {cap }}^{*}$ is determined to be,

$$
Q_{\text {cap }}^{*}=h^{* 2} \frac{\partial h^{*}}{\partial z^{*}}=\frac{1}{3 z_{2}^{*}}
$$

Substituting 2.10 into the global mass balance 2.8 gives the zeroth order governing ODE

$$
\frac{1}{3 z_{2}^{*}}=\frac{\partial z_{2}^{*}}{\partial t^{*}}
$$

which is solved with the initial condition to give

$$
z_{2}^{*}=\left(1+\frac{2}{3} t^{*}\right)^{1 / 2}
$$

The primary qualitative observations from 2.10 are that the capillary flow rate is inversely proportional to the fluid column length and from 2.12 that the column length eventually increases with $t^{1 / 2}$ for $t^{*} \gg 1$. It is possible to specify a simpler 
boundary condition $z_{2}(0)=0$ and achieve the result

$$
z_{2}^{*}=\left(\frac{2}{3} t^{*}\right)^{1 / 2}
$$

for an arbitrary length scale. If solutions were later desired for non-zero boundary conditions such as $z_{2}^{*}(0)=1$ one could employ an offset on time

$$
z_{2}^{*}=\left[\frac{2}{3}\left(t^{*}+t_{0}^{*}\right)\right]^{1 / 2}
$$

and solve $z_{2}^{*}(0)=1$ for $t_{0}^{*}$ to recover 2.12. A solution such as 2.12 is compact, and indicative of the relatively simple solution forms that can be found when using Eq. 1.4 to compute boundary conditions near a bulk meniscus. Notice that the scaling captures the fluid properties $\sigma$ and $\mu$, local geometry $\theta$ and $\alpha$ and cross section shape.

\subsection{Arbitrary section area}

In this section the cross sectional area is not assumed constant, but is left as a general weak function of $z$. The goal is to find a solution for $z_{2}$ in terms of $A_{s}(z)$ so that the effects of possible $z$ variation in section area can be understood. To facilitate the solution a slightly different mass balance is used, namely

$$
\left.Q_{c a p}\right|_{z_{2}}=\left.\left(A_{s}-m A_{f}\right)\right|_{z_{2}} \frac{\partial z_{2}}{\partial t}
$$

where $A_{f}$ is the flow area in a single corner flow. This approach avoids a messy integral that results from computing the gas volume in a complex geometry, and no information is lost in doing so. Eq. 2.13 is derived by drawing an infinitesimal 
control volume around the bulk meniscus and balancing flow rates. The results of the previous section are recovered using this formulation. A possible drawback is that $Q_{\text {cap }}$ must now be evaluated at $z_{2}$ (as opposed to zero), but the previous analysis suggests that the zeroth order solution for $Q_{\text {cap }}$ does not vary with $z$. It is necessary for container section shapes to satisfy $R \propto A_{s}^{1 / 2}$ so that Eq. 1.4 can be used to compute boundary conditions for the corner flows. Without this restriction $H$ is a more complex function of $z$ and analytical solutions are not apparent. The scales chosen remain the same but the length scale is not defined because it is arbitrary if $z_{2}(0)=0$. The mass balance 2.13 then becomes

$$
\left.Q_{c a p}^{*}\right|_{z_{2}}=\left.A_{s}^{*}\right|_{z_{2}^{*}}(1-\beta) \frac{\partial z_{2}^{*}}{\partial t^{*}}
$$

which, along with the dimensionless boundary condition on $h$ at $z_{2}$

$$
h^{*}\left(z_{2}^{*}\right)=\left.A_{s}^{* 1 / 2}\right|_{z_{2}^{*}}
$$

reveals the added complexity of $z$-dependent section areas. Taking the zeroth order solution for $h$ with the boundary condition 2.14 provides a general expression for capillary flow rate

$$
Q_{c a p}^{*}=\frac{\left.A_{s}^{*}\right|_{z_{2}^{*}} ^{3 / 2}}{3 z_{2}^{*}} .
$$

Combining 2.15 with 2.13 and manipulating slightly yields a general implicit equation for $z_{2}^{*}$

$$
t^{*}=\int \frac{3 z_{2}^{*}}{\left.A_{s}^{* 1 / 2}\right|_{z_{2}^{*}}} d z_{2}^{*},
$$




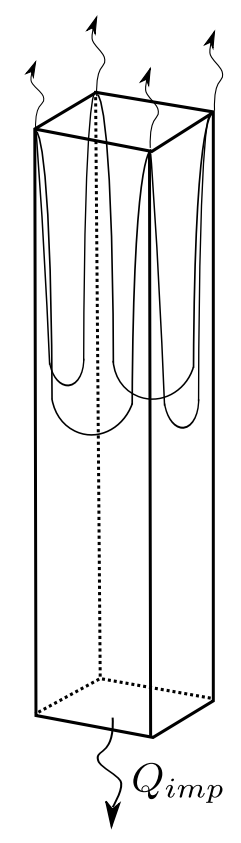

Figure 2.2: A 3-D sketch of a container being drained of liquid via capillary flows at the top as well as an 'imparted flow rate' $Q_{i m p}$ from the bottom.

which requires an initial condition following integration. Some dimensional solutions of Eq. 2.16 will be shown in Chapter 6.

\subsection{Imparted flow rates}

Container draining solutions are also possible with the addition of an imparted flow rate as depicted in Fig. 2.2. This is achieved by drawing fluid out of (or into) a container while simultaneously draining liquid via capillary corner flow. For simplicity, the imparted flow rate $Q_{i m p}$ is assumed constant and positive if drawing fluid from the container and negative if adding fluid to it. $Q_{i m p}$ appears only in the bulk mass balance as a forcing term at zeroth order, which is written 
dimensionally as

$$
Q_{c a p}+Q_{i m p}=A_{s} \frac{\partial z_{2}}{\partial t} .
$$

Several observations are noted from inspection of 2.17. First, for $Q_{i m p}<0$ (filling) a steady $z_{2}=$ constant solution is always possible in the limit $t \rightarrow \infty$ when the imparted flow rate fills as fast as the capillary flow rate drains. For such flows the length scale is not arbitrary and is determined by balancing the two terms on the LHS of 2.17 to find

$$
z_{s}=\frac{m \mathcal{W} F_{A} h_{s}^{3}}{Q_{i m p}} .
$$

Employing this scale the dimensionless governing differential equation for $z_{2}^{*}$ becomes

$$
\frac{1}{3 z_{2}^{*}} \pm 1=\frac{\partial z_{2}^{*}}{\partial t^{*}} .
$$

assuming $A_{s}^{*}=1$ and that the imparted flow rate is constant. The $(+)$ symbol is for positive (draining) and the (-) is for negative (filling) values of $Q_{i m p}$. The implicit solution to Eq. 2.18 is

$$
t^{*}= \pm z_{2}^{*}-\frac{\ln \left(1 \pm 3 z_{2}^{*}\right)}{3} .
$$

In Fig. 2.3, Eq. 2.19 is inverted and plotted for a positive and negative value of $Q_{i m p}$. Notice that for negative values of $Q_{i m p}$ the meniscus approaches a constant value $(1 / 3)$ such that the capillary flow rate out of the container is equal in magnitude to the imparted flow rate into the container. For positive values of $Q_{i m p}$ fluid is being withdrawn from the bottom of the container helping the downward bulk meniscus migration. In this case because the capillary flow rate decreases with $1 / z_{2}^{*}$ eventually the imparted flow rate will be much more significant than 


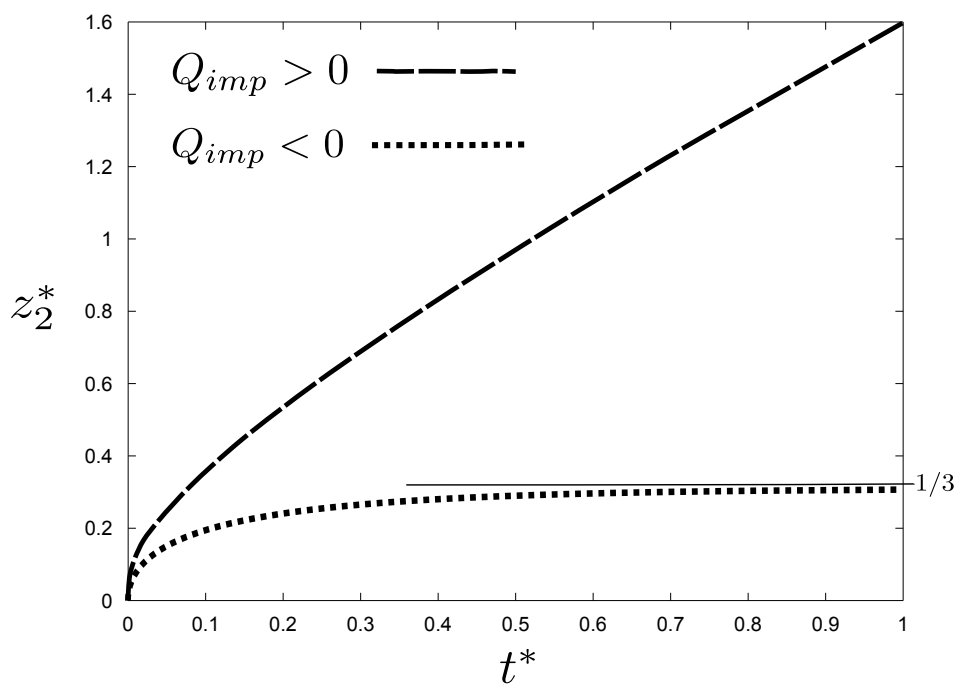

Figure 2.3: A container draining problem with constant section area and an imparted flow rate $Q_{i m p}$ out of the bottom of the container. $z_{2}^{*}$ is the dimensionless meniscus position. Notice that the meniscus eventually reaches a constant value $1 / 3$ for negative values of $Q_{i m p}$, which implies that fluid is being pumped into the bottom of the container.

the capillary flow and the container draining becomes relatively unaffected by the corner flows. The solution for $z_{2}$ then approaches a linear variation with time. 


\section{Chapter 3}

\section{Ullage migration solutions}

In this chapter ullage migration problems are studied. Again the capillary flow rate in the corners is found. A mass balance and initial condition are used to solve for the bulk meniscus position. Because two menisci are present in ullage migration problems, a volume constraint is used to relate the position of one bulk meniscus to another.

\subsection{Arbitrary section area}

A sample ullage migration problem is sketched in Fig. 3.1 for general $A_{s}^{*}$ where the assumptions used in the container draining model are already employed. The distance to the leftmost bulk meniscus is $z_{1}$, and $z_{2}$ to the rightmost. Eq. 1.4 is used to determine the known interface height at each bulk meniscus

$$
h\left(z_{1}\right)=\left.F_{H} A_{s}^{1 / 2}\right|_{z_{1}} \quad h\left(z_{2}\right)=\left.F_{H} A_{s}^{1 / 2}\right|_{z_{2}} .
$$

The solution method is again to first solve Eq. 1.1 with the above boundary conditions for $Q_{c a p}$, then substitute the results into a mass balance and solve for $z_{1}$ and $z_{2}$. If $\beta \ll 1$ the zeroth order mass balance at $z_{1}$ is

$$
\left.Q_{c a p}\right|_{z_{1}}=\left.A_{s}\right|_{z_{1}} \frac{\partial z_{1}}{\partial t}
$$




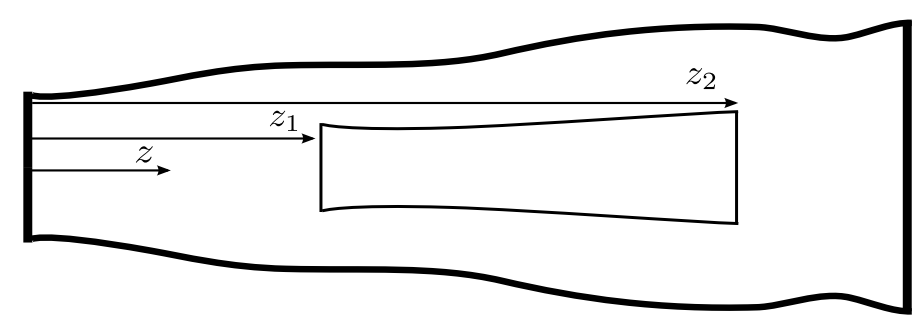

Figure 3.1: An ullage migration problem model with an arbitrary section area. The distance from the smallest section end to the first meniscus is $z_{1}$ and the distance to the larger meniscus is $z_{2}$. Capillary flows pump fluid from $z_{1}$ to $z_{2}$, displacing the gas ullage rightward. Note that the flat bulk meniscus assumption is employed in this model.

and a volume constraint is used to relate $z_{1}$ to $z_{2}$

$$
V_{u}=\int_{z_{1}}^{z_{2}}\left(A_{s}-m F_{A} h^{2}\right) d z
$$

where $V_{u}$ is the volume of the gas ullage. Choosing a length scale based on the volume constraint, $z_{s}=V_{u} / A_{0}$ the dimensionless zeroth order solution for $Q_{c a p}$ with the bulk meniscus balance is

$$
\frac{\left.A_{s}^{* 3 / 2}\right|_{z_{2}^{*}}-\left.A_{s}^{* 3 / 2}\right|_{z_{1}^{*}}}{3\left(z_{2}^{*}-z_{1}^{*}\right)}=A_{s}^{*} \frac{d z_{1}^{*}}{d t}
$$

and the volume constraint is

$$
1=\int_{z_{1}^{*}}^{z_{2}^{*}} A_{s}^{*} d z^{*},
$$

which requires $\beta h^{* 2} \ll A_{s}^{*}$, easily met for many containers. At this point it is awkward and perhaps confusing to continue with the general geometry $A_{s}$, so $A_{s}^{*}=z^{* 2}$ is chosen and solved as an example. Eq. 3.2 with the volume constraint 
is reduced to only one unknown

$$
\frac{1}{\left(3+z_{1}^{* 3}\right)-z_{1}^{*}}=z_{1}^{* 2} \frac{\partial z_{1}^{*}}{\partial t^{*}}
$$

and has the solution [6]

$$
t^{*}=\frac{1}{4}\left(\left(3+z_{1}^{* 3}\right)^{4 / 3}-z_{1}^{* 4}-3^{4 / 3}\right)
$$

An interesting geometry is one in which $A_{s}^{*}=z^{* 2 / 3}$ and Eq. 3.2 shows that the capillary flow rate is then constant, $Q_{\text {cap }}^{*}=m / 3$. In this unique geometry, $H$ increases as well as the viscous length $z_{2}-z_{1}$ such that the effects of each are canceled. Other solutions are shown in Table 5.1.

\subsection{Step reduction in area}

It is possible to apply the general equations derived in the previous section to containers with a step reduction in area, but a slightly different coordinate system shown in Fig. 3.2 allows for a cleaner solution and that is the reason why the solution is presented here. By analogy, this geometry is to an ullage migration problem as the constant section area geometry is to a container draining problem because the boundary conditions for the local flow $H_{1}$ and $H_{2}$ are constant. The larger section area is $A_{s 2}$ and the smaller one is $A_{s 1}$. Cross sectional geometry must be chosen such that each corner transitions smoothly from one side of the step to the other. The corner flow boundary conditions for these flows have constant values

$$
h\left(z_{1}\right)=H_{1}, \quad h\left(z_{2}\right)=H_{2} .
$$




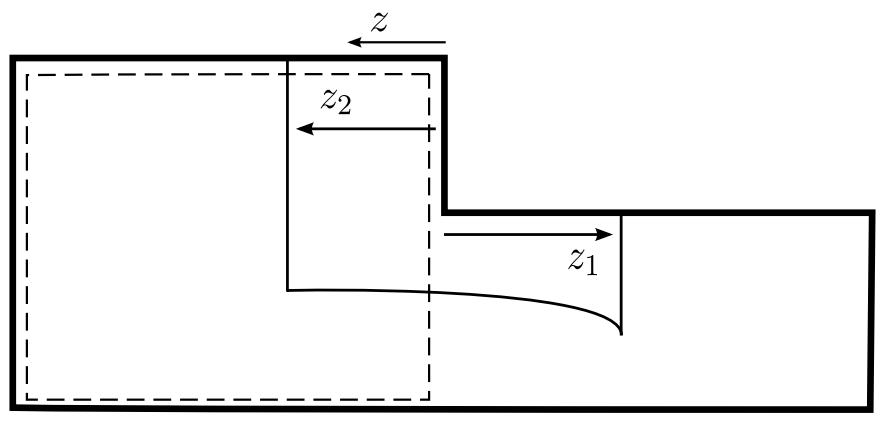

Figure 3.2: An ullage migration problem in a geometry possessing a step reduction in area. Capillary flows pump fluid from the right to the left, displacing the ullage leftward. The dotted line contains the control volume referred to in the text.

The scaling is based on the larger cross section,

$$
z_{s}=\frac{V_{u}}{A_{2}}, \quad t_{s}=\frac{A_{2} z_{s}^{2}}{m \mathcal{W} F_{A} H_{2}^{3}}, \quad h_{s}=H_{2},
$$

and the volume constraint is

$$
1=z_{2}^{*}+\Lambda z_{1}^{*}-\beta \int_{z_{1}}^{z_{2}^{*}} h^{* 2} d z
$$

where $\Lambda \equiv A_{s 1} / A_{s 2}$. With $\beta \ll 1$ the zeroth order flow rate is found to be

$$
Q^{*}=\frac{1-b^{3}}{3\left(\frac{1-z_{2}^{*}}{\Lambda}+z_{2}^{*}\right)}
$$

Combining 3.5 with the global mass balance 3.4 , a single zeroth order governing equation for a stepped cylinder is

$$
\frac{1-b^{3}}{3\left(\frac{1-z_{2}^{*}}{\Lambda}+z_{2}^{*}\right)}=\frac{\partial z_{2}^{*}}{\partial t^{*}}
$$


which may be solved to find

$$
z_{2}^{*}=\frac{\left[1-\frac{2}{3} t^{*} \Lambda(1-\Lambda)\left(1-b^{3}\right)\right]^{1 / 2}}{1-\Lambda}
$$

for $0 \leq \Lambda \leq 1$. The volume constraint can then be used to find

$$
z_{1} *=\left(1-z_{2}^{*}\right) / \Lambda
$$

In the limit $\Lambda \rightarrow 1$ a linear solution is recovered from 3.7

$$
z_{2}^{*}=\frac{\left(1-b^{3}\right) t}{3}
$$

and for the common situation $b^{3} \ll 1,3.7$ simplifies to

$$
z_{2}^{*}=\frac{\left[1-\frac{2}{3} t^{*} \Lambda(1-\Lambda)\right]^{1 / 2}}{1-\Lambda}
$$

For these stepped containers the flow will stop when $z_{2} \approx 1$ at time $t^{*}=t_{f}^{*}$ because then $H_{1}=H_{2}$ and no pressure gradient exists between the bulk menisci.

\subsection{Imparted flow rates}

In Fig. 3.3 fluid is pumped into the stepped section on the left side and withdrawn from the right side. The stepped geometry is chosen and analyzed here as an example, but the methods could be applied to any tapering or expanding sections. If in Fig. 3.3 the imparted flow rate is equal to the capillary flow rate in the corners the ullage does not move. If $Q_{i m p}$ was decreased slightly the ullage shortens, migrates left and the capillary flow rates increase. The ullage continues 


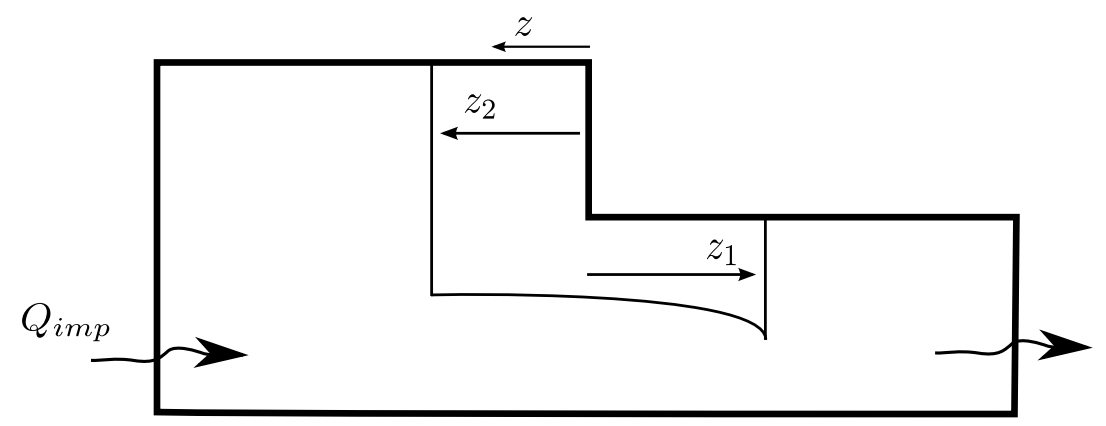

Figure 3.3: An ullage migration problem in a container with a step reduction in area and a flow rate being imparted into the left of the container and withdrawn from the right.

to move but is slowed by the imparted flow rate. If instead $Q_{i m p}$ is increased slightly the ullage moves right, lengthens and capillary flow rates decrease. The ullage continues to move rightward and capillary flow rates decrease due to the increased viscous resistance. It follows that it may be difficult to achieve a steady ullage position with a constant imparted flow rate and that the length scale is independent of $Q_{i m p}$. To solve for $z_{2}$ a control volume around the larger section is drawn and a mass balance is employed stating that the rate of change of fluid volume in the section with time is equal to the difference between capillary and imparted flow rates. The scaling is not altered in the presence of the imparted flow rate so the dimensionless mass balance is

$$
\frac{1}{3\left(\frac{1-z_{2}^{*}}{\Lambda}+z_{2}^{*}\right)}-Q_{i m p}^{*}=\frac{\partial z_{2}^{*}}{\partial t^{*}}
$$


where it is assumed that $b^{3} \ll 1$ and $Q_{i m p}^{*} \equiv \frac{Q_{i m p} z_{s}}{m \mathcal{W} F_{A} H_{2}^{3}}$. Notice that equation 3.6 is recovered if $Q_{i m p}=0$. The implicit solution to 3.9 is

$$
t^{*}=C_{1}-\frac{z_{2}^{*}}{Q_{i m p}^{*}}-\frac{\Lambda \ln \left[3 Q_{i m p}^{*}\left(1-z_{2}^{*}+\frac{\Lambda z_{2}^{*}}{3}\right)-\Lambda\right]}{(\Lambda-3) Q_{i m p}^{* 2}},
$$

where $C_{1}$ is determined based on the initial condition, chosen as $z_{2}(0)=1 / 2$ so that

$$
C_{1}=\frac{\Lambda \ln \left[\frac{1}{2}\left(\Lambda\left(Q_{i m p}^{*}-2\right)+3 Q_{i m p}^{*}\right)\right]}{Q_{i m p}^{* 2}(\Lambda-3)}+\frac{1}{2 Q_{i m p}^{*}} .
$$

There are 4 primary solutions captured in 3.10 depending on the sign and value of $Q_{i m p}$, and all are plotted on Fig. 3.4 for $\Lambda=1 / 2$. The first solution is the steady solution in which $Q_{c a p}^{*}(0)=Q_{i m p}^{*}$, where $z_{2}^{*}=1$. As mentioned above this may be difficult to maintain with constant values of $Q_{i m p}^{*}$. A second solution is found if $Q_{i m p}^{*}<0$ where the imparted flow rate speeds up the leftward ullage migration. A third is $0<Q_{i m p}^{*}<Q_{\text {cap }}^{*}(0)$ where the imparted flow rate works to slow down the leftward ullage migration but does not overcome it. The fourth solution is found if $0<Q_{c a p}^{*}<Q_{i m p}^{*}(0)$ and the imparted flow rate is greater than the capillary flow rate such that the ullage migration is reversed and only slowed by the capillary flow rate. 


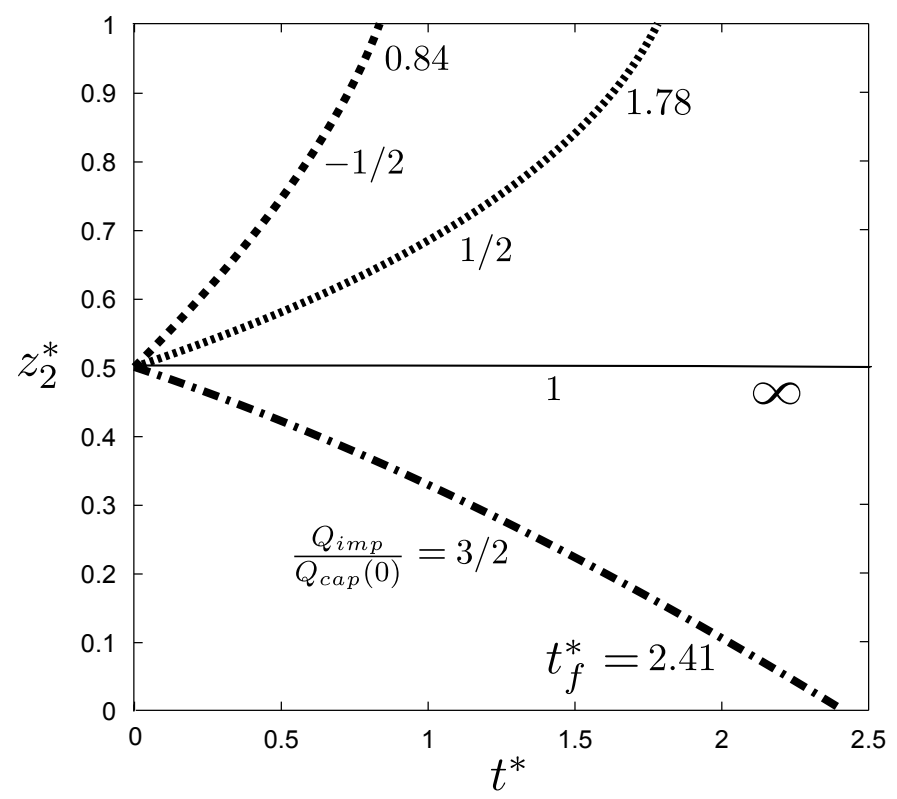

Figure 3.4: Ullage migration in a stepped container with an imposed flow rate for various values of $Q_{i m p} / Q_{c a p}(0)$, where $\Lambda \equiv A_{s 1} / A_{s 2}=1 / 2$ and $z_{2}(0)=1 / 2$ is assumed. The term $z_{2}^{*}$ is the distance from the step to the larger meniscus and $t_{f}^{*}$ is the time in it takes for the ullage to migrate entirely to one side of the step, such that no pressure gradient is present. 


\section{Chapter 4}

\section{Container draining problems with relaxed constraints}

In Chapter 2 container draining problems were studied and it was assumed that the cross section shape was such that $f(\theta, \alpha)$ was the same in every corner. In addition, only zeroth order solutions were found. In this chapter an $\mathcal{O}(\beta)$ solution is provided, and a system of equations governing the bulk meniscus position is provided that does not assume an 'equi- $f$ ' cross sectional shape.

\subsection{Irregular cross sections}

Irregular sections are defined here as sections where $f(\theta, \alpha)$ is not the same in every corner, implying that $H$ has a different value in each corner because $H=$ $R / f$. Each local corner flow must then be analyzed individually. For simplicity the cross section is assumed to be constant so $H$ is not $z$-dependent. The local corner flow equation applies in each of the $m$ wetted corners and a different scaling is used in each of them because the boundary conditions are potentially different in each corner. The boundary conditions at the bulk meniscus are

$$
h_{s j}=H_{2 j}
$$

where $H_{2 j}=R / f_{j}$ is the constant height value in the $j_{t h}$ of $m$ corners satisfying the Concus-Finn condition. The same time scale is chosen for every corner flow 
equation and for the bulk meniscus mass balance $t_{s}=A_{s} z_{s} / Q_{s}$, where

$$
Q_{s}=\sum_{j=1}^{m} F_{A j} H_{2 j}^{2} W_{j} \quad \mathcal{W}_{j} \equiv \frac{\sigma}{\mu}\langle\bar{w}\rangle^{*} \frac{\sin ^{2} \alpha_{j}}{f_{j}} \quad W_{j}=\mathcal{W}_{j} \frac{H_{2 j}}{z_{s}}
$$

and $z_{s}$ is again chosen to be $V(0) / A_{0}$. Some of the geometric functions defined previously in Chapter 2 require the $j$ subscript as well and are rewritten here for completeness:

$$
f_{j}=\frac{\sin \alpha_{j}}{\cos \theta-\sin \alpha_{j}} \quad F_{A j}=f_{j}^{2}\left(\frac{\cos \theta \sin \delta_{j}}{\sin \alpha_{j}}-\delta_{j}\right) .
$$

The $j_{t h}$ of $m$ edge flow equations is written in dimensionless form as

$$
\sum_{j=1}^{m} \beta_{j} \omega_{j} \frac{\partial h_{j}^{* 2}}{\partial t^{*}}=\omega_{j} \frac{\partial}{\partial z^{*}}\left(h_{j}^{* 2} \frac{\partial h_{j}^{*}}{\partial z^{*}}\right)
$$

The boundary condition at $z=0$ is still $h_{j}^{*}\left(0, t^{*}\right)=0$ for every corner, but individual boundary conditions must be applied in each corner at the bulk meniscus, $h_{j}^{*}\left(z_{2}^{*}\right)=H_{j}^{*}$. The two parameters appearing in 4.3 are

$$
\beta_{j}=\frac{A_{j}}{A_{s}}, \quad \omega_{j}=\frac{W_{j}}{W_{m}},
$$

where $W_{m}$ is the maximum of the $m$ local average velocity scales. The quantity $\sum_{j=1}^{m} \beta_{j} \omega_{j}$ appearing on the LHS of eq. 4.3 is again a ratio of total edge flow to bulk meniscus flow time scales. Appearing alone, $\beta_{j}$ is the ratio of the $j$ th edge flow to the bulk meniscus time scale. The condition $\sum_{j=1}^{m} \beta_{j} \omega_{j} \ll 1$ is required to satisfy the quasi-steady flow assumption. The parameter $\omega_{j}$ is a ratio of the $j$ th velocity scale to the maximum velocity scale, thus $0<\omega_{j} \leq 1$. When $\omega_{j} \ll 1$ 
the $j$ th corner flow does not contribute significantly to the overall flow rate and may be ignored at $\mathcal{O}(1)$. Using the scales chosen above, the dimensionless mass balance is

$$
\frac{\left.\sum_{j=1}^{m} \beta_{j} \omega_{j} h_{j}^{* 2} \frac{\partial h_{j}^{*}}{\partial z^{*}}\right|_{0}}{\sum_{j=1}^{m} \beta_{j} \omega_{j}}=\frac{\partial}{\partial t^{*}} \int_{0}^{z_{2}^{*}}\left(1-\sum_{j=1}^{m} \beta_{j} h_{j}^{* 2}\right) d z^{*}
$$

subject to the known initial volume condition

$$
z_{2}^{*}(0)=1+\int_{z_{1}^{*}}^{z_{2}^{*}} \sum_{j=1}^{m} \beta_{j} h_{j}^{* 2} d z^{*}
$$

The limiting case of $\beta_{j} \ll 1$ represents flows of low saturation and the liquid volume occupying the corner may be ignored at zeroth order in Eq. 4.5. The parameter $\beta_{j} \omega_{j} / \sum_{j=1}^{m} \beta_{j} \omega_{j}$ appears in each of the $m$ terms on the LHS of eq. 4.5 and represents the ratio of the $j$ th edge flow rate to total flow rate. With these equations derived the same methods used in Chapter 2 can then be applied to solve for $z_{2}$ accounting for irregular sections. It can be seen here that for geometries with identical cross flow area and velocity scales in each corner, $\beta_{j}=\omega_{j}=1$ and the system derived in Chapter 2 is recovered.

\section{2. $\mathcal{O}(\beta)$ solution}

Restricting again to constant section areas it is possible to extend earlier solutions from Chapter 2 to $\mathcal{O}(\beta)$. Expansions (commonly referred to as 'naïve' expansions) of the form

$$
h^{*}=h_{0}^{*}+\beta h_{1}^{*}+\mathcal{O}\left(\beta^{2}\right)
$$




$$
z_{2}^{*}=z_{20}^{*}+\beta z_{21}^{*}+\mathcal{O}\left(\beta^{2}\right)
$$

are substituted into the dimensionless system of equations 4.3 and 4.5 with the boundary conditions to give the $\mathcal{O}(1)$ system shown and solved in Chapter 2 and the first order system shown here where the $\mathcal{O}(\beta)$ corner flow equation is given by

$$
\frac{\partial h_{0}^{* 2}}{\partial t^{*}}=\frac{\partial}{\partial z^{*}}\left(2 h_{1}^{*} h_{0}^{*} \frac{\partial h_{0}^{*}}{\partial z^{*}}+h_{0}^{* 2} \frac{\partial h_{1}^{*}}{\partial z^{*}}\right)
$$

subject to boundary conditions $h_{1}^{*}\left(z_{11}^{*}\right)=0$ and

$$
h_{1}^{*}\left(z_{21}^{*}\right)=-\left.z_{21}^{*} \frac{\partial h_{0}^{*}}{\partial z^{*}}\right|_{z_{20}^{*}} .
$$

The $\mathcal{O}(\beta)$ mass balance is

$$
\left.\left(2 h_{1}^{*} h_{0}^{*} \frac{\partial h_{0}^{*}}{\partial z^{*}}+h_{0}^{* 2} \frac{\partial h_{1}^{*}}{\partial z^{*}}\right)\right|_{z^{*}=0}=\frac{\partial}{\partial t^{*}}\left(z_{21}^{*}-\int_{z_{10}^{*}}^{z_{20}^{*}} h_{0}^{* 2} d z^{*}\right)
$$

subject to the initial condition

$$
z_{21}^{*}(0)=\int_{0}^{z_{20}^{*}(0)} h_{0}^{* 2} d z^{*}
$$

Eq. 4.10 is determined at $\mathcal{O}(\beta)$ by direct substitution of the assumed solution forms 4.7 and 4.8 into Eq. 4.10

$$
h_{0}^{*}\left(z_{20}^{*}+\beta z_{21}^{*}+\mathcal{O}\left(\beta^{2}\right)\right)+\beta h_{1}^{*}\left(z_{20}^{*}+\beta z_{21}^{*}+\mathcal{O}\left(\beta^{2}\right)\right)+\mathcal{O}\left(\beta^{2}\right)=1,
$$


which is expanded via Taylor series to get

$$
h_{0}^{*}\left(z_{20}^{*}\right)+\beta h_{1}^{*}\left(z_{21}\right)+\left.\beta z_{21}^{*} \frac{\partial h_{0}^{*}}{\partial z^{*}}\right|_{z_{20}^{*}}+\mathcal{O}\left(\beta^{2}\right)=1
$$

The terms of 4.10 are now apparent in 4.13. Substituting the zeroth order result into the above system yields the following uniformly valid $\mathcal{O}(\beta)$ accurate solutions

$$
\begin{gathered}
h^{*}=\left(\frac{z^{*}}{z_{20}^{*}}\right)^{1 / 3}-\beta\left[\frac{1}{20}\left(\frac{z^{*}}{z_{20}^{*}}\right)^{1 / 3}-\left(\frac{z^{*}}{z_{20}^{*}}\right)^{2}\left(\frac{1}{20}+\frac{z_{21}^{*}}{3 z_{20}^{*}}\right)\right]+\mathcal{O}\left(\beta^{2}\right), \\
z_{2}^{*}=\left(1+\frac{2 t^{*}}{3}\right)^{1 / 2}+\frac{3 \beta}{20}\left[3\left(1+\frac{2 t^{*}}{3}\right)^{1 / 2}+1\right]+\mathcal{O}\left(\beta^{2}\right), \\
\left.Q^{*}\right|_{z=0}=\frac{1}{3 z_{20}^{*}}-\beta \frac{1}{20 z_{20}^{*}}+\mathcal{O}\left(\beta^{2}\right) .
\end{gathered}
$$

Notice that $z_{2}(0) \neq 1$ at $\mathcal{O}(\beta)$. By accounting for the fluid volume along the edge in the $\mathcal{O}(\beta)$ problem, $z_{2}^{*}(0)$ at $\mathcal{O}(\beta)$ is slightly greater than at $\mathcal{O}(1)$, thus $h^{*}$ must shift down so $h^{*}\left(z_{2}^{*}\right)=1$ and volume is conserved. The meniscus position $z_{2}$ then moves slightly quicker than predicted at zeroth order. Again it is possible to specify other boundary conditions such as $z_{2}(0)=z_{2 i}$. In this case it makes most sense to choose this known initial length as a length scale so that $z_{20}^{*}(0)=1$ and $z_{21}^{*}(0)=0$, giving the solution

$$
z_{2}^{*}=\left(1+\frac{2 t^{*}}{3}\right)^{1 / 2}+\frac{9 \beta}{20}\left[\left(1+\frac{2 t^{*}}{3}\right)^{1 / 2}-1\right]+\mathcal{O}\left(\beta^{2}\right)
$$

Notice that $z_{2}(0)=1$ at $\mathcal{O}(\beta)$. This initial condition eliminates the need to calculate the initial length as was required when initial volume is specified in 
Eq. 4.12. Another possible boundary condition is $z_{2}^{*}(0)=0$ which gives the result

$$
z_{2}^{*}=\left(\frac{2 t^{*}}{3}\right)^{1 / 2}\left(1+\frac{9 \beta}{20}\right)+\mathcal{O}\left(\beta^{2}\right)
$$

The fact that these solutions are uniformly valid gives confidence that higher order solutions are not significantly different from zeroth order solutions as $t$ increases. One is now able to accurately analyze containers in which $\beta^{2} \ll 1$, as opposed to the more restrictive constraint $\beta \ll 1$ 


\section{Chapter 5}

\section{Quick reference guide}

\subsection{Dimensional solutions}

Table 5.1 contains dimensional solutions to all flows studied so far with a few additions. There are 11 new (the pyramidally shaped container/ullage migration solution was presented in [6]) analytical solutions to 3-D transient free surface flows. The figures on the left side of the table are used to define whether the solution is for a draining or ullage migration problem. The second column defines the cross sectional area, the third gives the capillary flow rates and the fourth gives the explicit or implicit solution for meniscus position. Table 5.2 gives dimensional volume constraints where applicable. All arrows in figures refer to constant imparted flow rates. In a few solutions the term $z_{s}$ appears and must be found depending on the taper of the container in question. The dimensionless function $F_{Q}$

$$
F_{Q}=\frac{m\langle\bar{w}\rangle^{*} F_{A} F_{H}^{3} \sin ^{2} \alpha}{f}
$$

is used where it is assumed that $R \propto A_{S}^{1 / 2}$ and $f$ is the same in each corner and $F_{Q}$ contains all of the effects of cross sectional shape on capillary flow rate. In section $5.2 F_{Q}$ is detailed for some sample geometries. These tables demonstrate the utility of employing an easy to calculate boundary condition near a bulk meniscus. For both the ullage migration and draining problems general solutions are given so that one could quickly find a new solution for any desired geometry meeting the modeling restraints, which are summarized in Chapter 7. 
The following list serves as a step by step guide detailing how one could use Table 5.1 to quickly gather dimensional results regarding any of the flows discussed in this work as well as some other user specific flows.

1. The flow type and container geometry in question are found on the table by finding the corresponding figure in the first column.

2. The specific definition of the cross section area $A_{s}$ is then identified in the second column.

3. The dimensional capillary flow rate $Q_{c a p}$ is next identified in the third column and may be simply computed by hand, calculator, or spreadsheet.

4. The solution for meniscus position as a function of time is identified in the fourth column and may be also be computed if desired.

If the flow in question is an ullage migration problem, Table 5.2 can be similarly used to find the dimensional volume constraints that relate one bulk meniscus position to the other. These computed flow characteristics may be utilized when analyzing existing capillary systems or serve as a design tool for advanced systems. For example, one can compute the time required to displace an unwanted bubble. 


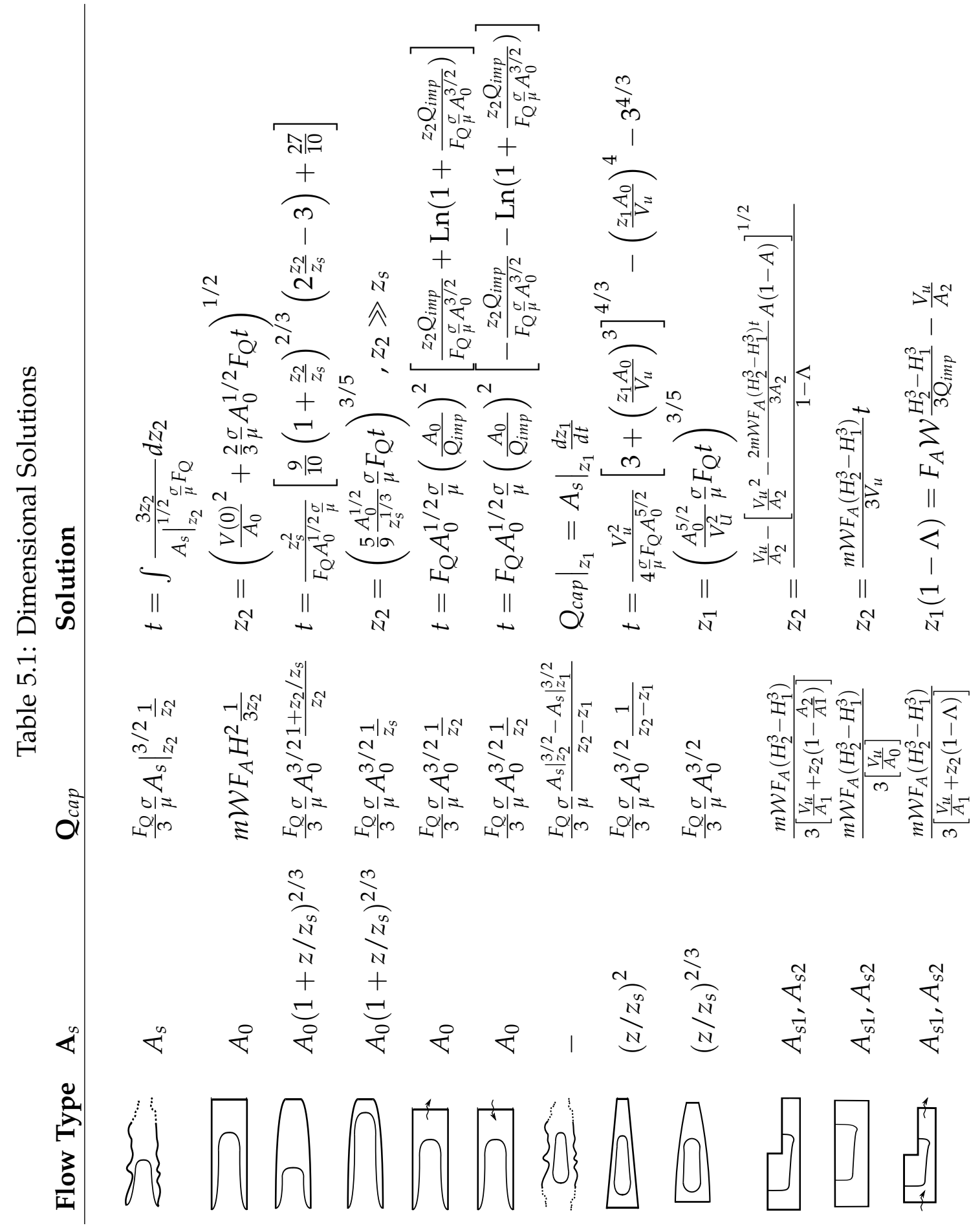


Table 5.2: Volume Constraints

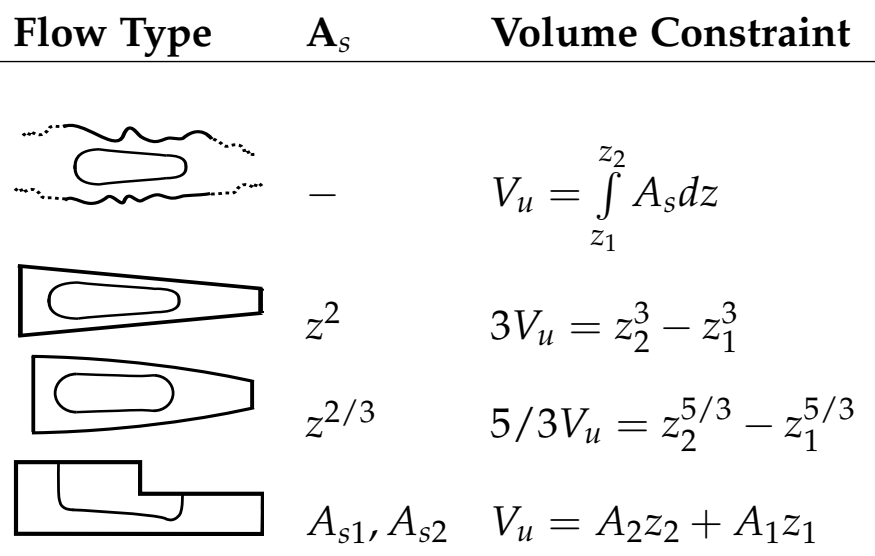

\subsection{Calculation of $F_{H}$ and $F_{Q}$ for some sample shapes}

It is apparent in Table 5.1 that the capillary flow rate $Q_{c a p}$ is linear with $F_{Q}$. Because $F_{Q}$ captures all of the cross sectional geometry it is then possible to compare different section shapes independent of total container capacity, section area $z$-dependence, and fluid properties. Below the functional forms for $F_{H}$ are reported in a few sample geometries and $F_{Q}$ is then maximized assuming a perfectly wetting fluid.

A regular polygonal cross section is a simple geometry for performance comparisons. The dimensionless function $F_{H}$ for a regular polygon is

$$
F_{H}=\frac{\cos \theta}{2 f \Sigma F_{N}^{1 / 2}}\left(1-\left(1-\frac{4 F_{N} \Sigma}{\cos ^{2} \theta}\right)^{1 / 2}\right)
$$

where

$$
F_{N}=\frac{1}{4 m \tan (\pi / m)}, \quad \Sigma=\frac{m F_{A}}{f^{2}} .
$$

$F_{N}$ is the ratio of section area to section perimeter and $\Sigma$ is the normalized flow area $A_{f} / R^{2}$. Considering only perfectly wetting fluids $F_{Q}$ is reduced to a function 


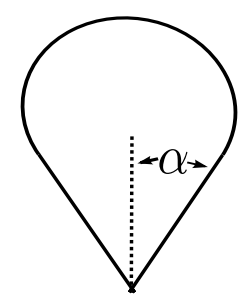

Figure 5.1: A snow cone section is a triangle with corner half angle $\alpha$ smoothly merged with a circular sector.

of $m$ only, which gives maximum flow rates when $m=3$, an equilateral triangle.

A single corner 'snow cone' geometry is shown in Fig. 5.1. The circular section meets the triangle smoothly with no slope discontinuity and Eq. 5.2 is used to calculate $F_{H}$ with

$$
F_{N}=\frac{\tan ^{2} \alpha\left[\frac{\pi}{2}-\alpha-\frac{\sin (\pi-2 \alpha)}{2}+\cos \alpha \sin \alpha\right]}{[(\pi-2 \alpha) \tan \alpha+2]^{2}}, \quad \Sigma=\frac{F_{A}}{f^{2}}
$$

Snow cone geometries maximize flow rates at $\alpha \approx 15^{\circ}$.

Some radially vaned container sections are shown in Fig. 5.2. Again $m$ is the number of corner flows and $n$ is the number of vanes with $m=2 n$ and $n \geq 2$. The vane length is related to the cylinder radius $r$ by a proportionality constant $\phi$ such that $\phi=v / r$ where $v$ is the vane length and

$$
F_{H}=\frac{v+\alpha}{\Sigma f \pi^{1 / 2}}\left[1-\left(1-\frac{\alpha \Sigma}{(v+\alpha)^{2}}\right)^{1 / 2}\right]
$$

where $\Sigma=\frac{F_{A}}{f^{2}}$ and is subject to the constraint $F_{H} \leq \frac{\pi^{1 / 2} \phi \sin \alpha}{f \sin \delta}$ to avoid pinning on the vanes [8]. The possibility of wetting occurring between the cylindrical tank 

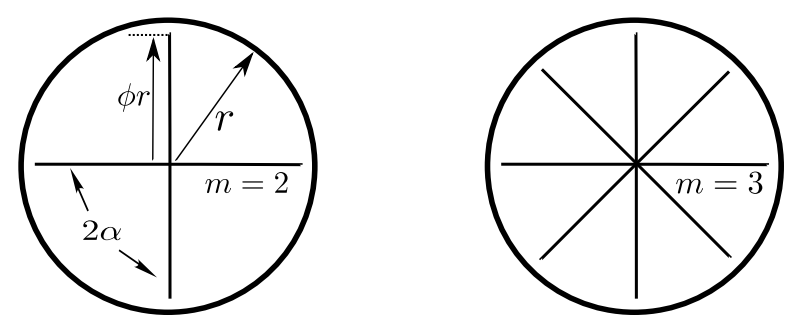

Figure 5.2: A radially vaned section is a circle with $m$ vanes in the center forming a corner angle $2 \alpha$. The vane length is related to the cylinder radius $r$ by a proportionality constant $\phi$ such that $\phi=v / r$ where $v$ is the vane length.

walls and the vanes is not considered currently, but has been observed in similar tank geometries [5]. The maximum flow rate is achieved with 13 vanes where $\phi \approx 0.823$ and $\alpha \approx 6.9^{\circ}$. 


\section{Chapter 6}

\section{Experiments}

A container draining experiment was performed to validate the theoretical prediction qualitatively if not quantitatively. The test cell used is a constant cross sectional area square cylinder manufactured from acrylic with $A_{s}=0.7396$ $\mathrm{mm}^{2}$. The cell is sealed at one end and filled with polydimethylsiloxane (PDMS, $5 \mathrm{cs}$ silicone oil at $20^{\circ} \mathrm{C}$ : density $\rho=890 \mathrm{~kg} / \mathrm{m}^{3}$, surface tension $\sigma=0.018 \mathrm{~N} / \mathrm{m}$, viscosity $\mu=0.00445 \mathrm{~kg} / \mathrm{m} \cdot \mathrm{s}$, and contact angle $\theta=0$ ). Once filled a tissue is placed firmly against the open end of the cell. The liquid wicks from the test cell into the tissue and the meniscus recedes. After a short transient period the corner flows are established and pump fluid from the bulk along the corners into the tissue. The test cell rests on a nominally leveled table and a digital SLR camera is programmed to take images of the flow every 8 minutes, see Fig. 6.1. From the images it is possible to record the bulk meniscus position and compare to theoretical predictions given in Table 5.1, where $\langle\bar{w}\rangle^{*}=0.12757$ [24].

The results are shown in Fig. 6.2 with a maximum under-prediction of the data by $\approx 9 \%$ when using the zeroth order solution. This single experiment is presented to give some confidence in the solution method, and not as an exhaustive proof of validity. More experiments would need to be performed to establish a true estimate of experiment error. Regardless, a limited discussion is insightful and could help guide future experiments. First, under-prediction is unexpected. Consider this list of possible sources of error. 


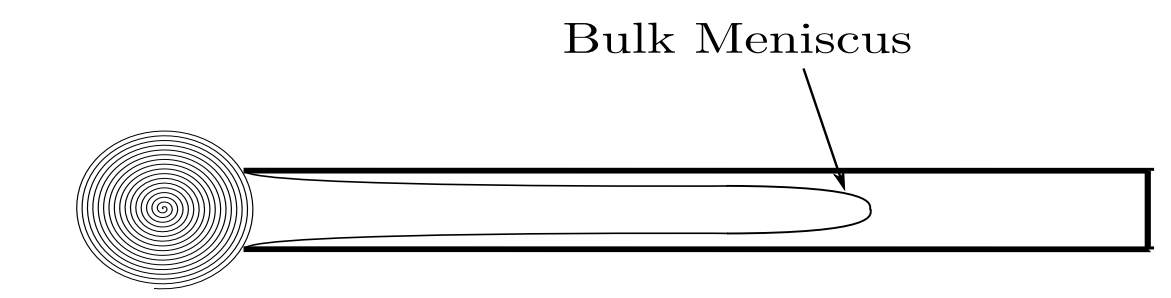

Tissue Roll

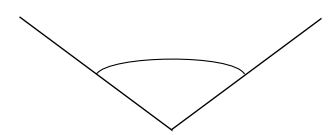

Camera

Figure 6.1: A square test cell $\left(A_{s}=0.7396 \mathrm{~mm}^{2}\right)$ is filled with polydimethylsiloxane (PDMS, $5 \mathrm{cs}$ silicone oil at $20^{\circ} \mathrm{C}$ : density $\rho=890 \mathrm{~kg} / \mathrm{m}^{3}$, surface tension $\sigma=0.018 \mathrm{~N} / \mathrm{m}$, viscosity $\mu=0.00445 \mathrm{~kg} / \mathrm{m} \cdot \mathrm{s}$, and contact angle $\theta=0$ ). A tissue roll is brought into contact with the open end, the bulk meniscus position is tracked using a digital camera.

1. The boundary condition at the tip is non-zero.

2. The corners are not sharp.

3. Inertia is significant.

4. The contact angle is not the static equilibrium angle.

5. The resistance to gas entering the container is significant.

6. The silicon oil was evaporating

7. The assumption $\beta \ll 1$ was not satisfied

8. The boundary condition at the bulk meniscus is wrong.

9. The effect of gravity is significant.

Notice that items $1-5$ would lead to over-prediction and are therefore not considered currently. Item 6 was accounted for by leaving a container full of the test fluid overnight and measuring the amount lost in the morning. The time 
scale for mass loss due to evaporation was orders of magnitude higher than than the time it took to run the experiment. Therefore evaporation is ruled out as a possible contributor to under-prediction. The parameter $\beta$ in this test is 0.06 and as a result the first order prediction given by Eq. 4.15 is $\approx 3 \%$ different than the zeroth order prediction, but still systematically underpredicts the experiment by $\approx 6.5 \%$. For these reasons item 7 is ruled out. The boundary condition at the bulk may have been wrong in two ways. First, it may have been applied in the wrong location. To investigate this potential source of error, the experiment is repeated using the same fluid but with thick-walled rounded square tubes (drawn quartz tube with $2.88 \mathrm{~mm}$ O.D., $1.02 \mathrm{~mm}$ rounded square, $0.12 \mathrm{~mm}$ corner radius, and $200 \mathrm{~mm}$ length). These tubes were chosen to reduce optical distortion and images of the bulk meniscus are captured through a microscope. For these long duration experiments the impact of tube level and precision, tissue resistance to air ingestion, tissue saturation, particulate contamination, and the effects of temperature dependent fluid properties including evaporation are all considered and demonstrated to be within the constraints of the modeling assumptions. Bulk meniscus interface profiles are taken from the images, then plotted in Fig. 6.3 where they are scaled and shifted by $\left(z-z_{b}\right) /\left(1+2 t^{* 1 / 2} / 3\right)^{1 / 2}+1 \mathrm{~mm}$. As can be seen the bulk region becomes smaller with respect to $z_{2}$ as time increases, giving confidence in the assumption $z_{H} \approx z_{b} \equiv z_{2}$. A similar experiment performed in [18] predicted these results based on similar but much shorter duration experiments. The value of the boundary condition might also be a source of error, but based on other works that used this same boundary condition and still over-predicted the experiments, item 8 does not seem to be a likely reason for the 
error. The effects of gravity are captured in the bond number $B o=\rho g R L \sin \phi / \sigma$ [22] where $L \approx z_{2}$ and $\phi$ is the tilt angle. Error due to gravity would then be expected to increase with $z_{2}$ for non-zero tilt angles. Note that gravity could cause either over-prediction or under-prediction due to an additional term in the local governing Eq. [22] which accounts for a body force and depends on the tilt angle $\phi$. Fig. 6.4 shows the experimental error as increasing with time. For these reasons it is suggested that this experiment was under-predicted by a maximum of $\approx 9 \%$ due to improper leveling of the experiment and item 9 is chosen as the most likely contributor to experimental error. 


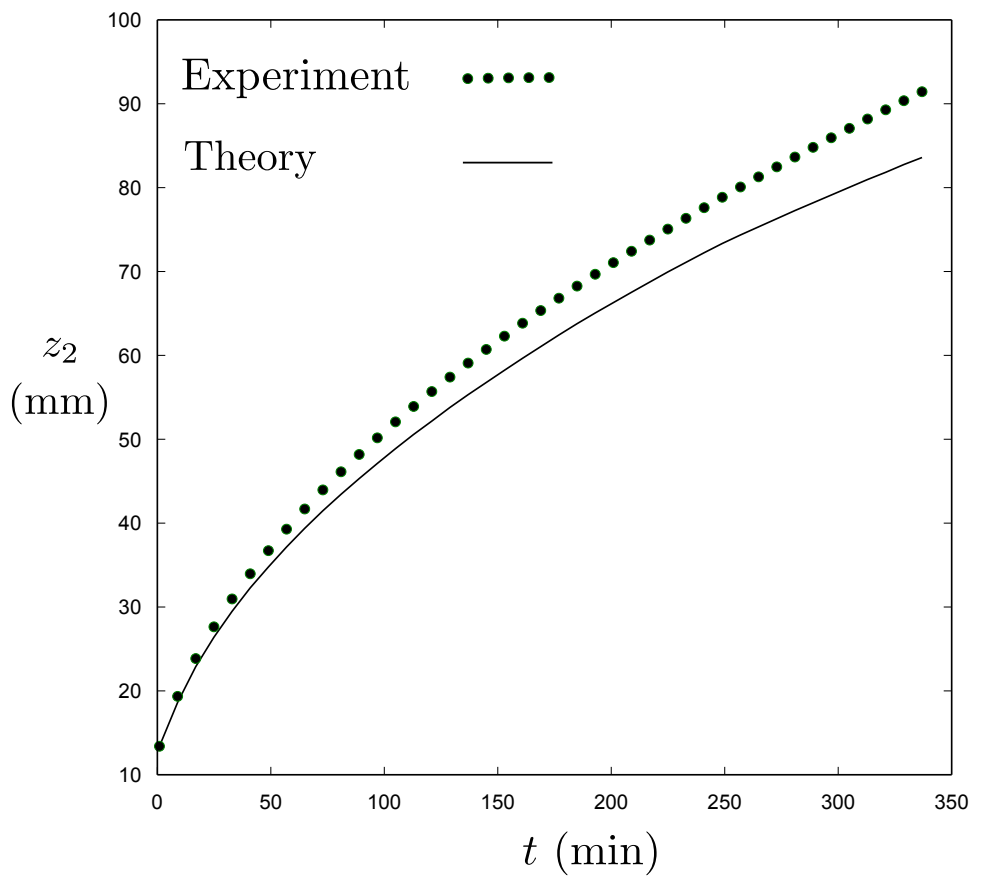

Figure 6.2: Comparison of experiment versus theory for a constant section area draining flow. $z_{2}$ is the distance from the container outlet to the bulk meniscus and $t$ is time. Notice the theory underpredicted the experimental results by $\approx 9 \%$, presumably because gravity effects were significant in the experiment and not accounted for in the theory. 


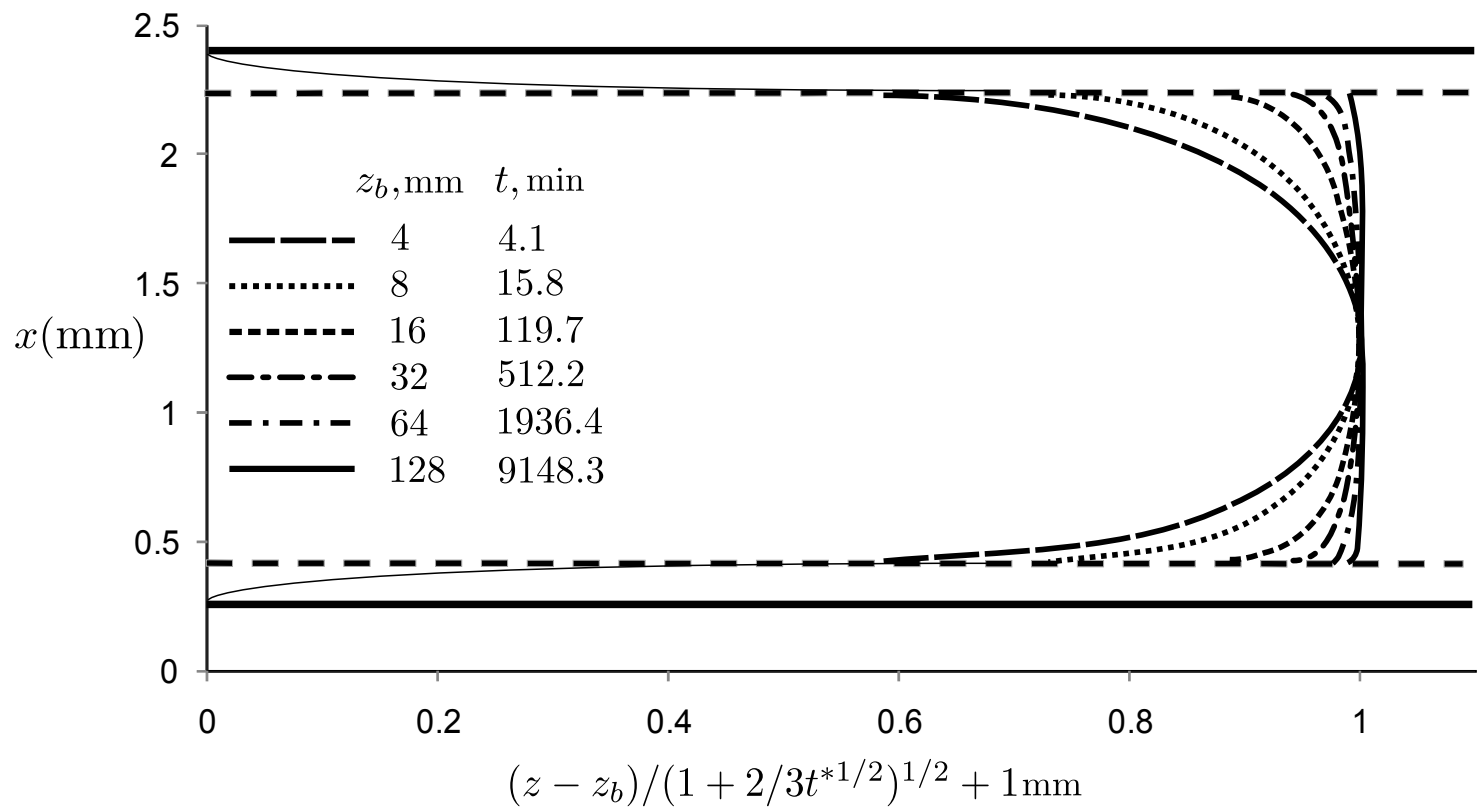

Figure 6.3: Bulk meniscus profiles scaled by corner flow length and shifted for comparison. The constant height $H$ is sketched as the thick dotted line. Notice that as time proceeds the size of the bulk region compared to the corner flow length diminishes, giving confidence in the flat bulk meniscus assumption. 


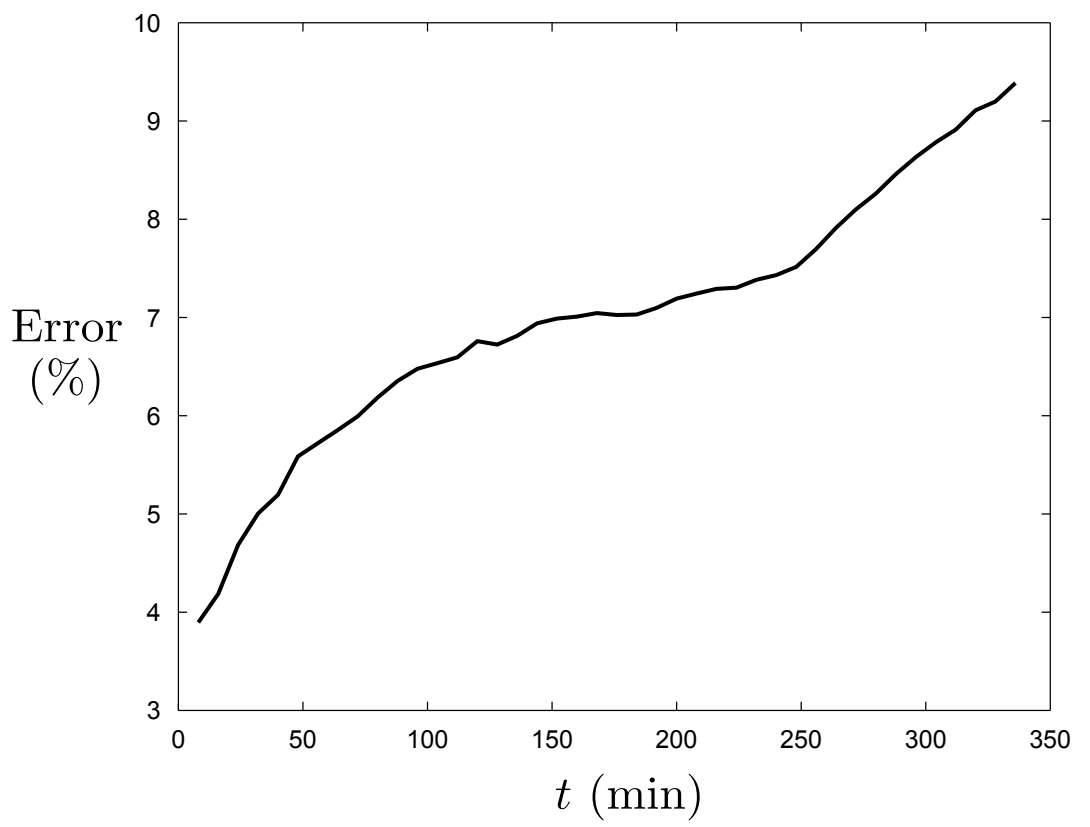

Figure 6.4: Experimental error in the single draining experiment plotted against time $t$. Error increases with time, and therefore with the length of the corner flow, suggesting that gravity effects are the most likely cause of underprediction reported in Fig. 6.2. 


\section{Chapter 7}

\section{Conclusion}

This thesis adds to the collection of solutions for capillary corner flows [27]. The governing PDE Eq. 1.1 used throughout is applicable for sharp corners with symmetric wetting conditions in zero gravity, but other equations governing the local flow (such as that of a rounded corner) could be utilized to find interface profiles and flow rates as well. The global geometry determines boundary conditions for Eq. 1.1 and a family of these geometries were presented and studied, all of which satisfy a quasi-steady assumption. These geometries, whether they are ullage migration or draining problems must meet the following criteria:

- $\beta \ll 1$. For a constant cross section draining problem an $\mathcal{O}(\beta)$ solution was found so this constraint could be relaxed to $\beta^{2} \ll 1$. It was noticed that the $\mathcal{O}(\beta)$ solution is not significantly different from the $\mathcal{O}(1)$ solution so it might be possible to accurately solve problems in which $\beta \approx 0.1$ or slightly larger. Future experiments could leverage this idea.

- $A_{s} / P_{s}^{2}$ must not be $z$-dependent. This is automatically satisfied for constant section areas. All regular polygons, snow cones, rhombi and the radially vaned container discussed above also meet this criteria even if $A_{s}$ is $z$ dependent. Rectangles, parallelograms, trapezoids and other irregular shapes can not be directly analyzed with these methods, if the section areas are $z$-dependent. 
- If the section area is not constant then it must be a weak function of $z$ where $\left|\partial \mathcal{S} / \partial z^{\prime}\right|^{2} \ll 1$ with $S$ being the distance from the center of the container to a given corner vertex.

Sample draining and ullage migration solutions were found for 10 sample global geometries and listed in Table 5.1. Also given in Table 5.1 are general solutions that can be used to determine solutions in geometries not explicitly studied here, for example a linearly varying section area such that $A_{s}=A_{0}\left(z / z_{s}\right)$ or perhaps a section in which $A_{s}=A_{0}\left(z / z_{s}\right)^{1 / 2}$. In all cases the methods of de Lazzer et al. detailed in [25] were used to compute boundary conditions for the local flow problem near an assumed flat bulk meniscus. The flat bulk meniscus assumption was validated with an experiment presented in Chapter 6. In general the closed form solutions are fairly simple, yielding explicit solutions in ullage migration problems with $A_{s} \propto z^{2 / 3}$, in the constant section draining problem, and for all stepped containers.

It was shown that cross sectional shapes can be intelligently selected to maximize flow rates, and sample shapes were presented. Different container tapers are also easily investigated from the solutions, and geometry selections can be made to give desired functional forms of bulk meniscus positions such as the typical $t^{1 / 2}$ dependence, linear dependence, natural log dependence, or other power laws and polynomials. Container tapers in which flow rates are constant independent of meniscus position are possible for tapers in which $A_{s} \propto z^{2 / 3}$.

Although the flows studied are idealized, it is possible to use the results in real world problems. For example the dependence of cross section shape on capillary flow rate was clearly identified throughout Chapter 5 in terms of the dimensionless function $F_{Q}$, see Table 5.1. In a recent work on heat pipe design 
it was determined experimentally that the critical heat input was increased by $\approx 1.8$ when switching from a rectangle to an equilateral triangle [11]. The triangular section had a much smaller section area though, so when normalizing by the section areas the ratio of critical heat input in a triangular section over the rectangular section is 8.28. Through $F_{Q}$ it is predicted that for isothermal draining flows the capillary flow rate in these sections would be increased by a factor of 6.86 when making the same geometry switch. Because it was identified that the available capillary pumping capacity has a significant effect on the critical heat input [7], $F_{Q}$ can be used to analyze other sections for heat pipe design. Also, in [8] possible cross sections were analyzed to reduce settling times of vaned containers in spacecraft. The same method for computing boundary conditions at the bulk was used, but the flow studied was that of an 'advancing tip' problem, where the corner flows have yet to reach the top of the container. It was shown that if using a radially vaned cross section then 12 corners minimized the time it would take for the corner flows to reach the top of the container, but no consideration was made as to the settling time after the top of the container was reached. In chapter 5 it is shown that 26 corner flows would reduce the settling time under these conditions. Three dimensional effects can also be investigated from the general dimensional solutions. For example if a container expands from an outlet with $z^{2 / 3}$ power it is evident that capillary flow rates are the same regardless of the fill level of the container. In the end designers in several fields have another tool set available to design capillary systems, and analysts could use the solutions to benchmark their numerical simulations or compare to experiments. 


\subsection{Future work}

The most obvious next step is an experimental work focused on validating the many solutions presented here. There are currently a suite of experiments prepared for flight to the International Space Station that will be analyzed primarily to confirm that the methods used to compute boundary conditions for local flows near bulk menisci are valid. Also, a fully numerical study has yet to be performed for any of the flows analyzed here. As mentioned above the analytic solutions could be used as a benchmark for the full numerical studies. If the solutions are shown to be consistent with experimental observation and numerical studies then engineers could confidently employ these analytic solutions, either in future experiments or when designing capillary systems.

Future analytical work on isothermal capillary corner flows should address some practical concerns. For example, water is commonly used as a working fluid in some capillary systems. Because contact angle hysteresis is common with water, it would be useful and potentially significant to determine how it affects capillary systems. A similar study could be performed to investigate the possibility of using capillary corner flows to passively separate phases. This would be especially useful in space applications, as passive systems are often significantly less expensive than active ones. Other studies could analyze pinning of the contact line as well as dynamic contact angle effects.

Specifically related to this work it is possible that some inspired choices in container geometry could lead to higher order solutions. A higher order solution is especially desirable in ullage migration problems, as one has yet to be found using asymptotic methods. If found the restraint $\beta \ll 1$ could be relaxed to $\beta^{2} \ll$ 
1, expanding the set of possible container geometries that could be analyzed. Also, the contribution of the terms that were neglected at zeroth order could be investigated and hopefully shown to be small for all time as was the case for the constant section draining solutions. 


\section{References}

[1] D. J. Chato. Cryogenic fluid transfer for exploration. Technical Report TM-215286, Lewis Research Center, Cleveland, OH, 2008.

[2] D. J. Chato. Low gravity issues of deep space refueling. Technical Report TM-213640, 2005.

[3] S. V Szabo, W. A. Groesbeck Jr., K. W. Baud, A. J. Stofan, T. W. Porada, and F. C. Yeh. Atlas-centaur flight ac-4 coast-phase propellant and vehicle behavior. Technical Report TM-1189, 1965.

[4] P. Concus and R. Finn. On the behavior of a capillary surface in a wedge. Proceedings of the National Academy of Sciences of the United States of America, 63(2):292-299, 1969.

[5] N. Tavan. Critical geometric wetting phenomena: study of capillary driven flow in the cfe vane-gap experiment aboard the international space station. Master's thesis, Portland State University, 2009.

[6] M.M. Weislogel, R. Jenson, and D. A. Bolleddula. Capillary driven flows in weakly 3-dimensional polygonal containers. volume AIAA Aerospace Sciences Meeting 13, pages 9260 - 9272, Reno, NV, United States, 2007.

[7] C. B. Sobhan, R. L. Rag, and G. P. Peterson. A review and comparative study of the investigations on micro heat pipes. International Journal of Energy Research, 31:664:688, 2007.

[8] M.M. Weislogel and S.H. Collicott. Capillary rewetting of vaned containers: Spacecraft tank rewetting following thrust resettling. AIAA Journal, 42(12):2551 - 2561, 2004.

[9] E. A. Thomas M. M. Weislogel and J. C. Graf. A novel device addressing design challenges for passive fluid phase separations aboard spacecraft. Microgravity Science and Technology, 21(3):257-268, 2009.

[10] J Zhang, S.J. Watson, and H. Wong. Fluid flow and heat transfer in a dual-wet micro heat pipe. Journal of Fluid Mechanics, 589:1-31, 2007.

[11] B. Sumana and P. Kumar. An analytical model for fluid flow and heat transfer in a micro-heat pipe of polygonal shape. International Journal of Heat and Mass Transfer, 58, 2005. 
[12] F. Goldschmidtboeing, M. Rabold, and P. Woias. Strategies for void-free liquid filling of micro cavities. Jounral of Micromechanics and Microengineering, 16, 2006.

[13] X. G. Yang F. Y. Zhang and C. Y. Wang. Liquid water removal from a polymer electrolyte fuel cell. Journal of The Electrochemical Society, 153, 2006.

[14] Z Dengen, M. Blunt, and F. M. ORR JR. Hydrocarbon drainage along corners of noncircular capillaries. Journal of Colloid and Interface Science, 187:11-21, 1997.

[15] Chen Y, Melvin L. S III, M. M. Weislogel, R.M. Jenson, S. Dhuey, and P.F. Nealey. Design, fabrication, and testing of microporous wicking structure. Microelectronic Engineering, 85, 2008.

[16] P. S. Ayyaswamy, I. Catton, and D. K. Edwards. Capillary flow in triangular grooves. Journal of Applied Mechanics, Transactions ASME, 41(4b):332, 1974.

[17] T. C. Ransohoff and C. J. Radke. Laminar flow of a wetting liquid along the corners of a predominantly gas-occupied noncircular pore. Journal of Colloid and Interface Science, 121(392), 1988.

[18] M.M. Weislogel and S. Lichter. Capillary flow in an interior corner. Journal of Fluid Mechanics, 373:349 - 378, 1998.

[19] M.M. Weislogel, Y Chen, and D Bolledulla. A better nondimensionalization scheme for slender laminar flows: The Laplacian operator scaling method. Physics of Fluids, 20(2):163 - 170, 2008.

[20] B. Semerjian. Capillary flow in interconnected corners and other exciting adventures. Master's thesis, Portland State University, 2009.

[21] Y. Chen, M. M. Weislogel, and C. Nardin. Capillary-driven flows along rounded interior corners. Journal of Fluid Mechanics, 566:235 - 271, 2006.

[22] E. Ramé and M.M. Weislogel. Gravity effects on capillary flows in sharp corners. Physics of Fluids, 21(4):042106, 2009.

[23] C. Nardin. Computation of capillary flow in interior corners: Dissimilarly wetted corners and rounded corners. Master's thesis, Portland State University, 2005.

[24] D. Bolleddula. Capillary flow analysis and computation of de-wetting and wetting resistances in angular geometries. Master's thesis, Portland State University, 2007. 
[25] A. de Lazzer, D. Langbein, M. Dreyer, and H.J. Rath. Mean curvature of liquid surfaces in cylindrical containers of arbitrary cross-section. Microgravity Science and Technology, 9(3):208 - 219, 1996.

[26] M.M. Weislogel. Capillary flow in containers of polygonal section. AIAA Journal, 39(12):2320 - 2326, 2001.

[27] M.M. Weislogel. Some analytical tools for fluids management in space: Isothermal capillary flows along interior corners. Advances in Space Research, 32(2):163 - 170, 2003. 